\title{
Next-to-leading order unitarity fits in Two-Higgs-Doublet models with soft $\mathbb{Z}_{2}$ breaking
}

\author{
Vincenzo Cacchio, ${ }^{a}$ Debtosh Chowdhury, ${ }^{a}$ Otto Eberhardt ${ }^{a}$ and \\ Christopher W. Murphy ${ }^{b}$ \\ ${ }^{a}$ Istituto Nazionale di Fisica Nucleare, Sezione di Roma, \\ Piazzale Aldo Moro 2, I-00185 Roma, Italy \\ ${ }^{b}$ Scuola Normale Superiore, \\ Piazza dei Cavalieri 7, I-56126 Pisa, Italy \\ E-mail: vincenzo.cacchio@roma1.infn.it, \\ debtosh.chowdhury@roma1.infn.it, otto. eberhardt@roma1.infn.it, \\ christopher.murphy@sns.it
}

ABstract: We fit the next-to-leading order unitarity conditions to the Two-Higgs-Doublet model with a softly broken $\mathbb{Z}_{2}$ symmetry. In doing so, we alleviate the existing uncertainty on how to treat higher order corrections to quartic couplings of its Higgs potential. A simplified approach to implementing the next-to-leading order unitarity conditions is presented. These new bounds are then combined with all other relevant constraints, including the complete set of LHC Run I data. The upper $95 \%$ bounds we find are 4.2 on the absolute values of the quartic couplings, and $235 \mathrm{GeV}(100 \mathrm{GeV})$ for the mass degeneracies between the heavy Higgs particles in the type I (type II) scenario. In type II, we exclude an unbroken $\mathbb{Z}_{2}$ symmetry with a probability of $95 \%$. All fits are performed using the open-source code HEPfit.

Keywords: Beyond Standard Model, Higgs Physics

ARXIV EPRINT: 1609.01290 


\section{Contents}

1 Introduction 1

2 Model 3

3 Constraints 4

3.1 Theoretical constraints 4

$\begin{array}{ll}3.2 & \text { Experimental constraints }\end{array}$

$\begin{array}{llr}4 & \text { HEPfit } & 8\end{array}$

5 Results $\quad 9$

6 Conclusions $\quad 15$

$\begin{array}{lr}\text { A Additional figures } & 18\end{array}$

B Diagonal entries of the NLO $S$-matrix 24

$\begin{array}{ll}\text { C Experimental inputs } & 26\end{array}$

\section{Introduction}

Run I of the Large Hadron Collider (LHC) has concluded with the discovery of the last missing piece of the Standard Model (SM) - the Higgs boson [1, 2]. It has tested the validity of the SM in a previously unexplored regime of energy, and has not found any significant deviations from the SM, hinting at a gap in the mass spectrum from the SM to whatever lies beyond it. This picture is consistent with the absence of indications of New Physics (NP) coming from indirect searches (e.g. electroweak precision or flavour observables). Certainly Run I of the LHC did not address all the shortcomings of the SM - among which are the hierarchy problem, dark matter and an explanation for the flavour pattern. In order to find a solution for these problems the presence of NP is inevitable. One of the key questions for Run II of the LHC is then at what scale the NP appears. The measured value of the Higgs mass, $m_{h} \approx 125 \mathrm{GeV}[3]$, hints at an answer to this: it tells us that the Higgs potential of the SM is not stable up to very high energy scales $[4,5]$. Thus one has to introduce an additional mechanism or new degrees of freedom to stabilize the Higgs potential if one wants to exclude the possibility of vacuum metastability. Furthermore, to keep the SM Higgs mass natural, new degrees of freedom around the $\mathrm{TeV}$ scale are required [6].

One well motivated direction for discovering physics beyond the SM is to search for additional Higgs bosons. These particles often arise in natural theories of electroweak 
symmetry breaking, e.g. the Higgs sector of the minimal supersymmetric Standard Model (MSSM) [7-9], twin Higgs models [10-14], composite Higgs models [15, 16]. Also, there is no fundamental reason for the minimality of the SM scalar sector, and multiple generations are known to exist in the fermion sector. Furthermore, the uncertainties in the SM Higgs coupling measurement [17] do not exclude the presence of additional scalars.

After the SM, the simplest and most straightforward extension of the SM is the addition of another Higgs doublet, the so-called Two-Higgs-Doublet model (2HDM) [18-20], which has been analysed in great detail in the literature, see for instance [21-47]. It is interesting to study the unitarity bounds in the $2 \mathrm{HDM}$ because the scale at which new particles are expected to appear based on naturalness arguments is the same scale as the Lee, Quigg, Thacker upper limit $[48,49]$ on the Higgs mass in the SM, which is of order of $1 \mathrm{TeV}$. In fact, there exists a large number of works studying the tree-level unitarity bounds on the quartic couplings, $\lambda_{i}$, and Higgs masses of the 2HDM, see e.g. [50-56]. Unlike in the SM, extracting the bounds on the masses of the Higgs boson from the bounds on the quartic couplings is not straightforward because in the $2 \mathrm{HDM}$ the quartic couplings are in general functions of more parameters than just the masses of the Higgs bosons and their corresponding vacuum expectation values (VEVs). Recently, the perturbative unitarity bounds in the $\mathcal{C P}$-conserving softly-broken $\mathbb{Z}_{2}$ symmetric $2 \mathrm{HDM}$ were analyzed at the one-loop level [57]. This calculation settled a particular issue regarding how to estimate higher-order effects on available upper limits on the quartic couplings: in the SM, the unitarity bounds had been determined beyond the leading order (LO), and the typical result was that the bounds on the (RG-improved) quartic coupling of the SM were improved by a factor of a few with respect to the tree-level analysis [58-61]. While it was known that the tree-level unitarity bounds in NP models were likely to be overly conservative, there was no well defined way to implement stricter bounds using only tree-level results. Inspired by the SM results, it was advocated to re-scale the tree-level conditions by a factor of $1 / 4$ to estimate higher order contributions [34]. A renormalization group analysis at next-to-leading order (NLO) confirmed this prescription if one wants a stable Higgs potential beyond $10 \mathrm{TeV}$ [44]. However, now that an explicit NLO computation is at hand, this uncertainty on how to treat higher order corrections to the partial-wave amplitudes has been removed.

In this article, we improve on the results currently available in the literature in two main ways: regarding the unitary constraints, we go beyond the leading order precision by employing one-loop corrections which are enhanced, $\mathcal{O}\left(\lambda_{i} \lambda_{j} / 16 \pi^{2}\right)$, in the limit $s \gg$ $\left|\lambda_{i}\right| v^{2} \gg M_{W}^{2}, s \gg m_{12}^{2}$ to all the $2 \rightarrow 2$ longitudinal vector boson and Higgs boson scattering amplitudes. Secondly, we perform global parameter fits including the most upto-date Run I ATLAS and CMS results, rather than only using a handful of benchmark scenarios, which might not cover the whole spectrum of interesting features.

The structure of this article is as follows: we give a short introduction to the model and its constraints in sections 2 and 3, respectively. The statistical framework is presented in section 4. Section 5 contains the results of the fits. We conclude in section 6 . Supplementary figures can be found in appendix A, while we list the formulae for the NLO unitarity criteria and the fit inputs in appendices B and C, respectively. 


\section{Model}

The two-Higgs-doublet model (2HDM) [18-20] is a simple and straightforward extension of the Standard Model (SM), obtained through the addition of a second Higgs doublet to the SM field content. A characteristic of general 2HDMs is the existence of flavour-changing neutral currents (FCNC) mediated by tree-level exchange of neutral Higgs bosons. A natural way to eliminate these potentially dangerous FCNC is to require that the Yukawa interactions respect a discrete $\mathbb{Z}_{2}$ symmetry, which can be broken softly. The $\mathbb{Z}_{2}$ symmetry can be chosen in four independent ways, depending on the $\mathbb{Z}_{2}$ charge assignments for quarks and charged leptons; this lead to four different types of $2 \mathrm{HDM}$ which are referred to as type I, type II, type X (lepton specific) and type Y (flipped). The type II model is of particular interest because the Higgs sector of the MSSM is a $2 \mathrm{HDM}$ of type II. The models we focus on in this paper are the $\mathcal{C} \mathcal{P}$-conserving $2 \mathrm{HDM}$ of type I and II with a softly broken $\mathbb{Z}_{2}$ symmetry. The most general Higgs potential in a $\mathcal{C P}$-conserving $2 \mathrm{HDM}$ with a softly broken $\mathbb{Z}_{2}$ symmetry reads

$$
\begin{aligned}
V= & m_{11}^{2} \Phi_{1}^{\dagger} \Phi_{1}+m_{22}^{2} \Phi_{2}^{\dagger} \Phi_{2}-m_{12}^{2}\left(\Phi_{1}^{\dagger} \Phi_{2}+\Phi_{2}^{\dagger} \Phi_{1}\right)+\frac{1}{2} \lambda_{1}\left(\Phi_{1}^{\dagger} \Phi_{1}\right)^{2}+\frac{1}{2} \lambda_{2}\left(\Phi_{2}^{\dagger} \Phi_{2}\right)^{2} \\
& +\lambda_{3}\left(\Phi_{1}^{\dagger} \Phi_{1}\right)\left(\Phi_{2}^{\dagger} \Phi_{2}\right)+\lambda_{4}\left(\Phi_{1}^{\dagger} \Phi_{2}\right)\left(\Phi_{2}^{\dagger} \Phi_{1}\right)+\frac{1}{2} \lambda_{5}\left[\left(\Phi_{1}^{\dagger} \Phi_{2}\right)^{2}+\left(\Phi_{2}^{\dagger} \Phi_{1}\right)^{2}\right]
\end{aligned}
$$

where $\Phi_{1}$ and $\Phi_{2}$ are the two complex $\mathrm{SU}(2)_{L}$ Higgs doublets with hypercharge $Y=1 / 2$ and the eight scalar potential parameters are real to avoid explicit $\mathcal{C} \mathcal{P}$-violation, with $m_{12}^{2}$ being the $\mathbb{Z}_{2}$ soft-breaking parameter. At the global minimum of the scalar potential $V$ the neutral components of $\Phi_{1}$ and $\Phi_{2}$ acquire VEVs, $v_{1} / \sqrt{2}$ and $v_{2} / \sqrt{2}$, respectively, which are fixed by the minimization of the scalar potential and must satisfy $v_{1}^{2}+v_{2}^{2} \equiv v^{2} \approx(246 \mathrm{GeV})^{2}$. The ratio of the two VEVs is defined as $\tan \beta=v_{2} / v_{1}$, where $0 \leq \beta \leq \pi / 2$. Assuming no $\mathcal{C P}$-violation in the Higgs sector, the physical scalar spectrum consists of two $\mathcal{C P}$-even states $h$ and $H$ with $m_{h}<m_{H}$, the $\mathcal{C P}$-odd state $A$ and the charged state $H^{ \pm}$. The masses of these scalar bosons are denoted as $m_{\phi}$ with $\phi \in\left\{h, H, A, H^{ \pm}\right\}$. Throughout this paper we interpret the observed Higgs resonance as the light $\mathcal{C P}$-even scalar $h$ and thus treat $m_{h}$ as fixed by measurements, $m_{h}=125.09 \mathrm{GeV}[3]$. We choose the independent physical parameters of the model to be

$$
\tan \beta, \quad \beta-\alpha, \quad m_{12}^{2}, \quad m_{H}, \quad m_{A}, \quad m_{H^{ \pm}},
$$

where $\alpha$ is the mixing angle of the neutral $\mathcal{C P}$-even 2HDM Higgs bosons. In this parametrization, the tree-level couplings of the Higgs bosons to vector bosons and fermions only depend on $\tan \beta$ and $\beta-\alpha$. Moreover, for $\beta-\alpha=\pi / 2$ the couplings of $h$ to SM fermions and vector bosons are SM-like and $H$ does not couple to vector bosons at treelevel; the literature refers to this as the alignment limit [19, 62-64].

Considering only the third generation of fermions, the Yukawa Lagrangian under the above-mentioned $\mathbb{Z}_{2}$ symmetry takes the following shape:

$$
\mathcal{L}_{Y}=-Y_{t} \bar{Q}_{L} i \sigma_{2} \Phi_{2}^{*} t_{R}-Y_{b} \bar{Q}_{L} \Phi_{k} b_{R}-Y_{\tau} \bar{L}_{L} \Phi_{k} \tau_{R}+\text { h.c. }
$$




\begin{tabular}{|l|c|c|c|c|}
\hline Parameter & $\tan \beta$ & $\beta-\alpha$ & $m_{12}^{2}$ & $m_{H}, m_{A}, m_{H^{ \pm}}$ \\
\hline Range & {$[0.25 ; 100]$} & {$[0 ; \pi]$} & {$\left[-5 \cdot 10^{4} ; 7 \cdot 10^{5}\right] \mathrm{GeV}^{2}$} & {$[130 ; 1100] \mathrm{GeV}$} \\
\hline
\end{tabular}

Table 1. Priors on the 2HDM parameters.

where the top quark couples to $\Phi_{2}$ by convention and the index $k$ is 2 in type I and 1 in type II. The top Yukawa coupling is related to the SM value $Y_{t}^{\mathrm{SM}}$ by $Y_{t}=Y_{t}^{\mathrm{SM}} / \sin \beta$, while $Y_{f}=Y_{f}^{\mathrm{SM}} / \sin \beta$ in type I and $Y_{f}=Y_{f}^{\mathrm{SM}} / \cos \beta$ in type II for $f=b, \tau$.

\section{Constraints}

In this section we list and discuss the theoretical and experimental constraints we impose on the $2 \mathrm{HDM}$ parameter space. Since we want to combine them in a Bayesian fit, we list the priors on the parameters from (2.2):

\subsection{Theoretical constraints}

On the theory side, constraints on the $2 \mathrm{HDM}$ come from the following requirements:

- the Higgs potential must be bounded from below [65] between $M_{Z}$ and $750 \mathrm{GeV}$,

- the minimum of the Higgs potential at $246 \mathrm{GeV}$ should be the global minimum [66],

- the 2HDM quartic couplings $\lambda_{i}(i=1,2,3,4,5)$ and the Yukawa couplings are assumed to be perturbative (i.e. smaller than $4 \pi$ and $\sqrt{4 \pi}$ in magnitude, respectively) at least up to $750 \mathrm{GeV}$,

- the $S$-matrix of $2 \rightarrow 2$ scattering processes for Higgs bosons and longitudinal vector bosons should be unitary up to NLO, and its NLO eigenvalues should not exceed the LO eigenvalues in magnitude [57].

Requiring positivity and perturbativity of the couplings to hold at least up to $750 \mathrm{GeV}$ is motivated by the fact that this scale is well above the electroweak symmetry breaking scale and we can safely use the NLO unitarity conditions. For the renormalization group running we use NLO renormalization group equations (RGE) [44].

The first three bullet points have already been used in the literature and will be referred to as "stability up to $750 \mathrm{GeV}$ " in the following. The fourth set of constraints has never been applied in a general $2 \mathrm{HDM}$ fit, which is why we want to explain the details of our approach in the following, referring to [57].

The unitarity of the $S$-matrix leads to constraints on the partial wave amplitudes of a theory,

$$
\left|a_{\ell}^{2 \rightarrow 2}-\frac{1}{2} i\right|^{2}+\sum_{n>2}\left|a_{\ell}^{2 \rightarrow n}\right|^{2}=\frac{1}{4},
$$

where $a_{\ell}^{2 \rightarrow n}$ are the eigenvalues of the matrix of the $\ell$-th, $2 \rightarrow n$ partial wave amplitudes, $\mathbf{a}_{\ell}$. Considering only $2 \rightarrow 2$ scattering (and dropping the superscript) this constraint becomes 
an inequality,

$$
\left|a_{\ell}-\frac{1}{2} i\right| \leq \frac{1}{2}
$$

The 2HDM one-loop corrections necessary to use this inequality were recently computed in ref. [57]. Prior to this computation, the inequalities $\left|\operatorname{Re}\left(a_{0}\right)\right| \leq \frac{1}{2}$ or $\left|a_{0}\right| \leq 1$ were used to constrain the tree-level partial wave amplitudes [50-56]. Comparing with the discussion of higher order corrections in the SM, stronger bounds were estimated and used for the 2HDM [34, 44], but this ansatz was controversial. Having at hand the calculated NLO unitarity conditions, we can determine the upper bound on the quartic couplings without any ambiguity of method.

The computation of ref. [57] was performed in the high energy limit, $s \gg\left|\lambda_{i}\right| v^{2} \gg M_{W}^{2}$, $s \gg m_{12}^{2}$, where the $\mathrm{SU}(2)_{L} \times \mathrm{U}(1)_{Y}$ symmetry is manifest. In this limit, $\mathbf{a}_{0}$ is block diagonal at leading order, with blocks of definite weak isospin $(\sigma)$ and hypercharge $(Y)$ $\left(\mathbf{a}_{\ell>0}=\mathbf{0}\right.$ at leading order in this limit). Furthermore, the $\mathbb{Z}_{2}$-even and -odd states do not mix at tree-level, leading to smaller blocks. Due to the manifest symmetry at high energies, the calculation can be simplified by computing the amplitudes in the $\mathbb{Z}_{2}$ basis using the non-physical Higgs fields, $w_{j}^{ \pm}, n_{j}^{(*)}[54]$,

$$
\Phi_{j}=\left(\begin{array}{c}
w_{j}^{+} \\
n_{j}+v_{j} / \sqrt{2}
\end{array}\right), \quad n_{j}=\frac{h_{j}+i z_{j}}{\sqrt{2}}, \quad(j=1,2) .
$$

The elements of $\mathbf{a}_{0}$ are given by

$$
\left(\mathbf{a}_{0}\right)_{i, f}=\frac{1}{16 \pi s} \int_{-s}^{0} d t \mathcal{M}_{i \otimes f}(s, t)
$$

where, for example,

$$
\begin{aligned}
\mathcal{M}_{\frac{1}{\sqrt{2}}\left(\Phi_{1}^{\dagger} \Phi_{1}\right) \otimes \frac{1}{\sqrt{2}}\left(\Phi_{2}^{\dagger} \tau^{3} \Phi_{2}\right)=\frac{1}{2}}\left(\mathcal{M}_{w_{1}^{+} w_{1}^{-} \rightarrow w_{2}^{+} w_{2}^{-}}-\mathcal{M}_{w_{1}^{+} w_{1}^{-} \rightarrow n_{2} n_{2}^{*}}\right. \\
\left.+\mathcal{M}_{n_{1} n_{1}^{*} \rightarrow w_{2}^{+} w_{2}^{-}}-\mathcal{M}_{n_{1} n_{1}^{*} \rightarrow n_{2} n_{2}^{*}}\right) .
\end{aligned}
$$

In general, the block diagonal structure of $\mathbf{a}_{0}$ does not hold beyond tree-level. However, it turns out that in the high energy limit, this structure is only broken by diagrams that correct the wavefunctions of the external legs, not by 1PI diagrams. Ref. [57] showed that the external wavefunction corrections are numerically subdominant with respect to the 1PI diagrams in some special cases. We confirm this and find it to be generalizable for all $2 \mathrm{HDM}$ scenarios with a softly broken $\mathbb{Z}_{2}$ symmetry. Due to this relative numerical unimportance, we neglect the external wavefunction corrections throughout this work. In 
this approximation, the one-loop eigenvalues take the following form, ${ }^{1}$

$$
\begin{aligned}
& 32 \pi a_{00 \pm}^{\text {even }}=B_{1}+B_{2} \pm \sqrt{\left(B_{1}-B_{2}\right)^{2}+4 B_{3}^{2}}, \\
& 32 \pi a_{00 \pm}^{\text {odd }}=2 B_{4} \pm 2 B_{6}, \\
& 32 \pi a_{01 \pm}^{\text {even }}=B_{7}+B_{8} \pm \sqrt{\left(B_{7}-B_{8}\right)^{2}+4 B_{9}^{2}}, \\
& 32 \pi a_{01 \pm}^{\text {odd }}=2 B_{13} \pm 2 B_{15}, \\
& 32 \pi a_{10}^{\text {odd }}=2 B_{19} \\
& 32 \pi a_{11 \pm}^{\text {even }}=B_{20}+B_{21} \pm \sqrt{\left(B_{20}-B_{21}\right)^{2}+4 B_{22}^{2}}, \\
& 32 \pi a_{11}^{\text {odd }}=2 B_{30},
\end{aligned}
$$

with the eigenvalues labeled as follows, $a_{Y \sigma \pm}^{\mathbb{Z}_{2}}$, and dropping the index $\ell=0 . \quad B_{N}$ is the block-diagonal element, $\left(\mathbf{a}_{0}\right)_{i, f}$, from eq. (B.N) in [57], which can also be found in appendix B. In order to satisfy unitarity, the $a_{Y \sigma \pm}^{\mathbb{Z}_{2}}$ have to individually fulfill the condition (3.2). Note that at LO, the eigenvalues are related to the ones defined in [54] by $a_{Y \sigma \pm}^{\mathbb{Z}_{2}}=-32 \pi^{2} \Lambda_{Y \sigma \mp}^{\mathbb{Z}_{2}}$ for $\lambda_{5}>0$.

Another constraint is the requirement that higher order corrections to the partial wave amplitudes are suppressed. In particular, following [57] we define,

$$
R_{1}^{\prime}=\frac{\left|a_{Y \sigma \pm}^{\mathbb{Z}_{2}, \mathrm{NLO}}\right|}{\left|a_{Y \sigma \pm}^{\mathbb{Z}_{2}, \mathrm{LO}}\right|},
$$

where the (N)LO label denotes the pure (N)LO contribution. Similar criteria were used in the perturbative unitarity analysis of the SM in ref. [60]. Assuming that the power series is perturbatively stable, we want to require the NLO contribution to be smaller than the LO expression, hence $R_{1}^{\prime}<1$. However, we need to avoid the exclusion of accidentally small leading-order contributions. (For instance, $a_{10}^{\text {odd,LO }}=\left(\lambda_{4}-\lambda_{3}\right) /(8 \pi)$ is small if $\lambda_{3} \approx \lambda_{4}$, while $a_{10}^{\text {odd,NLO }}$ also depends on the other quartic couplings.) Therefore, we decided to use the $R_{1}^{\prime}$ criterion only if $\left|a_{Y \sigma \pm}^{\mathbb{Z}_{2}, \mathrm{LO}}\right|>0.02 \approx 1 /(16 \pi)$.

The 2HDM is unitary, so let us explain what we mean when we say unitarity constraints. Inequality (3.2) requires the couplings of a theory to be smaller than a certain value in magnitude, or else the theory will no longer appear to be unitary at the finite order of the perturbative expansion to which we are working. In this sense both the "perturbativity bound," $R_{1}^{\prime}$, and the "unitarity bound" test the same thing, namely where the breakdown of perturbation theory occurs.

\subsection{Experimental constraints}

The experimental constraints included in our analysis are:

- the Peskin-Takeuchi parameters $S, T$, and $U$ [67],

\footnotetext{
${ }^{1}$ Ref. [57] used the differential operator $\mathcal{D}_{\mathrm{GMU}}=16 \pi^{2} \mu^{2}\left(d / d \mu^{2}\right)$ in its definition of the beta functions. In this work we use the traditional definition of the beta function, $\beta_{\lambda_{i}} \equiv \mathcal{D} \lambda_{i}=\mu\left(d \lambda_{i} / d \mu\right)$.
} 
- the $h$ signal strengths,

- the non-observation of $H$ and $A$ at the LHC and

- the $B_{s}$ meson mass difference $\Delta m_{B_{s}}[68,69]$ and the branching ratio $\mathcal{B}(\bar{B} \rightarrow$ $\left.X_{s} \gamma\right)[70]$.

As we saw in the previous section, the $2 \mathrm{HDMs}$ introduce new Higgs bosons which couple to the gauge bosons and which, thereby, can give contributions, through loops, to the gauge boson self-energies. Thus, the $2 \mathrm{HDM}$ yield new contributions to $S, T$ and $U$ that generally move them away from their SM values. For the 2HDM predictions of the Peskin-Takeuchi parameters in the $\mathcal{C P}$-conserving limit we make use of the formulae of [67]. As input values for the oblique parameters $S, T$ and $U$ and their correlation coefficients we take the most recent results obtained in a fit to electroweak precision data with HEPfit [71], see table 2 in appendix $\mathrm{C}$.

In order to confront the 2HDM with the latest ATLAS and CMS Run I data on Higgs signal strengths, we compute in the narrow-width approximation for each final state $f \in\{\gamma \gamma, Z Z, W W, b b, \tau \tau\}$ the signal strengths ${ }^{2}$

$$
\begin{aligned}
\mu_{\mathrm{ggF}+\mathrm{tth}}^{f} & =\sum_{i=\mathrm{ggF}, \mathrm{tth}} \frac{\sigma_{i}^{2 \mathrm{HDM}}}{\sigma_{i}^{\mathrm{SM}}} \cdot \frac{\mathcal{B}^{2 \mathrm{HDM}}(h \rightarrow f)}{\mathcal{B}^{\mathrm{SM}}(h \rightarrow f)}, \\
\mu_{\mathrm{VBF}+\mathrm{Vh}}^{f} & =\sum_{i=\mathrm{VBF}, \mathrm{Vh}} \frac{\sigma_{i}^{2 \mathrm{HDM}}}{\sigma_{i}^{\mathrm{SM}}} \cdot \frac{\mathcal{B}^{2 \mathrm{HDM}}(h \rightarrow f)}{\mathcal{B}^{\mathrm{SM}}(h \rightarrow f)},
\end{aligned}
$$

having grouped the Higgs production modes in just two effective modes, ggF + th and $\mathrm{VBF}+\mathrm{Vh}$, where "ggF", "tth", "VBF" and "Vh" stand for "gluon fusion", "tt $\bar{t}$ associated production", "vector boson fusion" and "Higgstrahlung", respectively. The SM Higgs boson production cross sections are taken at a centre-of-mass energy of $8 \mathrm{TeV}$ from [72]; the SM branching ratios were calculated with HDecay 6.10 [73]. In order to express the 2HDM cross sections and branching ratios in terms of the SM ones, we make use of the formulae of [74] for the loop induced decays of the neutral Higgs bosons. Central values, errors (Gaussian approximation) and correlations for the signal strengths in (3.8) and (3.9) were obtained from figure 13 and table 14 of [17] and can be found in table 3 in appendix C.

Direct $H$ and $A$ searches are taken into account as follows: given the $X \rightarrow H / A \rightarrow Y$ process, we define the ratio

$$
R_{\text {Gauss }}^{(X \rightarrow H / A \rightarrow Y)}=\frac{\left.\sigma \mathcal{B}\right|_{\text {theo }}-\left(\left.\sigma \mathcal{B}\right|_{95 \%, \text { obs }}-\left.\sigma \mathcal{B}\right|_{95 \%, \exp }\right)}{\left.\sigma \mathcal{B}\right|_{95 \%, \exp }},
$$

where $\sigma \mathcal{B}=\sigma(X \rightarrow H / A) \cdot \mathcal{B}(H / A \rightarrow Y)$ and the subscripts denote the theoretical $2 \mathrm{HDM}$ value of $\sigma \mathcal{B}$ and its observed and expected exclusion limit at $95 \% \mathrm{CL}$ by the experiments. With this definition, we can assume the $R_{\text {Gauss }}^{(X \rightarrow A \rightarrow Y)}$ ratios to be Gaussian with a standard deviation of 1 . Note that these quantities depend on $m_{H / A}$; furthermore we neglect the error on $\left.\sigma \mathcal{B}\right|_{95 \% \text {,exp }}$.

\footnotetext{
${ }^{2}$ For the sake of simplicity, we refrain from writing obvious antiparticle and charge attributions explicitly.
} 
The $H$ and $A$ search exclusion limits included in our analysis and their mass ranges, along with the exclusion plots from which they were digitalized, are listed in table 4 in appendix C. Most SM Higgs production cross sections are taken from the LHC Higgs Cross section Working Group [75]; the remaining ones are calculated with HIGLU 4.34 [76], Sushi 1.5 [77], and Madgraph5 2.2.2 [78]. The branching ratios were calculated with HDecay 6.10 [73] while the decay widths for both Higgs-to-Higgs decays and Higgs decays into a Higgs boson and a gauge boson are taken from [79].

From the plethora of flavour observables we only use the most relevant two for our 2HDM discussion: the mass difference in the $B_{s}$ meson system, $\Delta m_{B_{s}}$ and the branching fraction $\mathcal{B}\left(\bar{B} \rightarrow X_{s} \gamma\right)$. The former is calculated according to [68, 69] at LO. For the inclusive measurement of $\mathcal{B}\left(\bar{B} \rightarrow X_{s} \gamma\right)$, NNLO corrections are important [70]. As for fixed SM parameters this observable only depends on the two 2 HDM parameters $\tan \beta$ and $m_{H^{ \pm}}$, we store the $\mathcal{B}\left(\bar{B} \rightarrow X_{s} \gamma\right)$ values for various inputs of these two parameters in tables, and interpolate them linearly in the fits. A theoretical error of $7 \%$ is applied, which corresponds to the uncertainty in the SM parameters. The experimental inputs for the flavour observables can be found in table 5 in appendix C.

\section{HEPfit}

As numerical set-up we use the open-source code HEPfit [80], interfaced with the release version of the Bayesian Analysis Toolkit (BAT) [81]. The former calculates all mentioned 2HDM observables and feeds them into the parallelized BAT, which applies the Bayesian fit with Markov chain Monte Carlo simulations. The complete global fit with all theoretical bounds runs for approximately 60 hours with 12 parallel chains generating $2 \cdot 10^{7}$ iterations each. Adding the experimental observables as described above slows down the same fit to roughly 90 hours.

A fundamental difference between the Bayesian and the frequentist approach is the treatment of fine-tuning: if one changes the parametrization of a model, flat priors on the former parameters usually do not translate into flat distributions of the new basis in a Bayesian fit. Some values for a new parameter might only be obtained by a very specific constellation of the old parameters, which in that sense would mean that they require a certain amount of fine-tuning. A frequentist fit is not sensitive to this bias, but one could argue that it is also less natural. HEPfit makes use of the Bayesian approach assuming flat priors for the physical parameters (2.2), and the posterior distribution of the parameters in the Higgs potential (2.1) are "distorted" by the Jacobian of the change of parameters. However, the posterior intervals only have a well-defined meaning once experimental data is included, and that is when the dependence on the priors disappears. In the first part of the following section, when we only discuss theoretical constraints, the reader should bear in mind that our results depend on the priors (and thus on the parametrization). Also, we will present the $99.7 \%$ allowed regions for fits to only theoretical constraints, while after the inclusion of experimental data we show the $95.4 \%$ probability contours, which then have a statistical meaning. 


\section{Results}

In the following we will present the results of our fits of theoretical and experimental constraints to the $2 \mathrm{HDM}$ of type I and II. Before we address the physical $2 \mathrm{HDM}$ parameters we want to compare the effects of the unitarity constraints. As explained in section 3.1, we impose these bounds at a scale of $750 \mathrm{GeV}$; thus a stable Higgs potential at least up to this scale is implicitly assumed. Nonetheless, all quantities shown in the figures are to be understood at the scale $M_{Z}$.

In figure 1 we show the $99.7 \%$ probability regions for all $\lambda_{i}$ vs. $\lambda_{j}$ planes with three different unitarity conditions: the green areas are allowed if we impose only LO unitarity, the red regions show the remaining parameter space if we use the NLO unitarity conditions, and the blue contours result from additionally requiring the NLO unitarity conditions to be perturbative $\left(R_{1}^{\prime}<1\right)$. Generally one can see that perturbative NLO unitarity is a stronger constraint than NLO unitarity with arbitrary $R_{1}^{\prime}$, which itself is always stronger than LO unitarity. Numerically the largest possible absolute values for any of the quartic couplings from (2.1) are 8.10, 7.21, or 5.75, if we apply LO, NLO or perturbative NLO unitarity, respectively. Especially in the $\lambda_{4}$ vs. $\lambda_{3}$ plane, but also in the $\lambda_{5}$ vs. $\lambda_{3}$ and $\lambda_{5}$ vs. $\lambda_{4}$ planes one can see that for particular constellations, NLO unitarity features sharp incisions towards the origin of the plane, whereas those indentations are absent if we use LO unitarity.

A closer look at different variations of our conditions explained in section 3.1 is shown in the left panel of figure 2 in the $\lambda_{4}$ vs. $\lambda_{3}$ plane. The green, red and blue solid lines correspond to the contours of the same colour in the previous figure; all lines are the $99.7 \%$ probability boundaries. As explained above, previous studies used 1/4 rather than $1 / 2$ as upper limit for the real part of the LO unitarity eigenvalues. This choice is represented by the green dashed line and is almost always less stringent than $R_{1}^{\prime}$-perturbative NLO unitarity. The red dashed line uses LO RGE instead of the NLO RGE which apply in all other cases. As already stated in ref. [44], the NLO RGE "stabilize" the potential with respect to the LO expressions in the sense that for the same starting point one runs into non-perturbative values for the quartic couplings at much lower scales with LO RGE. That is why larger values for the $\lambda_{i}$ are accessible at the electroweak scale if one uses the NLO RGE. What happens if one only requires that $R_{1}^{\prime}<1$ without imposing NLO unitarity can be seen at the pink contour. In other words, the blue line should be interpreted as the combination of the red (NLO unitarity) and the pink one. Only for $\lambda_{3}>4$, one can see that the combination of both sets of constraints is stronger than their individual impacts. Finally, the cyan contour is the result of using a ten times smaller threshold for the LO part of $R_{1}^{\prime}$. However, compared to our typical limit of 0.02 this is not substantially different.

Again in the $\lambda_{4}$ vs. $\lambda_{3}$ plane, we also show the individual contributions of the relevant single NLO eigenvalues in the right panel of figure 2. The shaded areas are excluded at $99.7 \%$ probability by the eigenvalues indicated in the legend. It is worth noting that only "-" solutions seem to be important in this plane.

Going from the potential parametrisation to the physical parameters, one can see how the different constraints on the $\lambda_{i}$ couplings translate into restrictions on the mass differences between the heavy Higgs bosons $H, A$ and $H^{+}$in figure 3. Like in figure 1, we observe a hierarchy between LO unitarity, NLO unitarity and NLO unitarity with the 

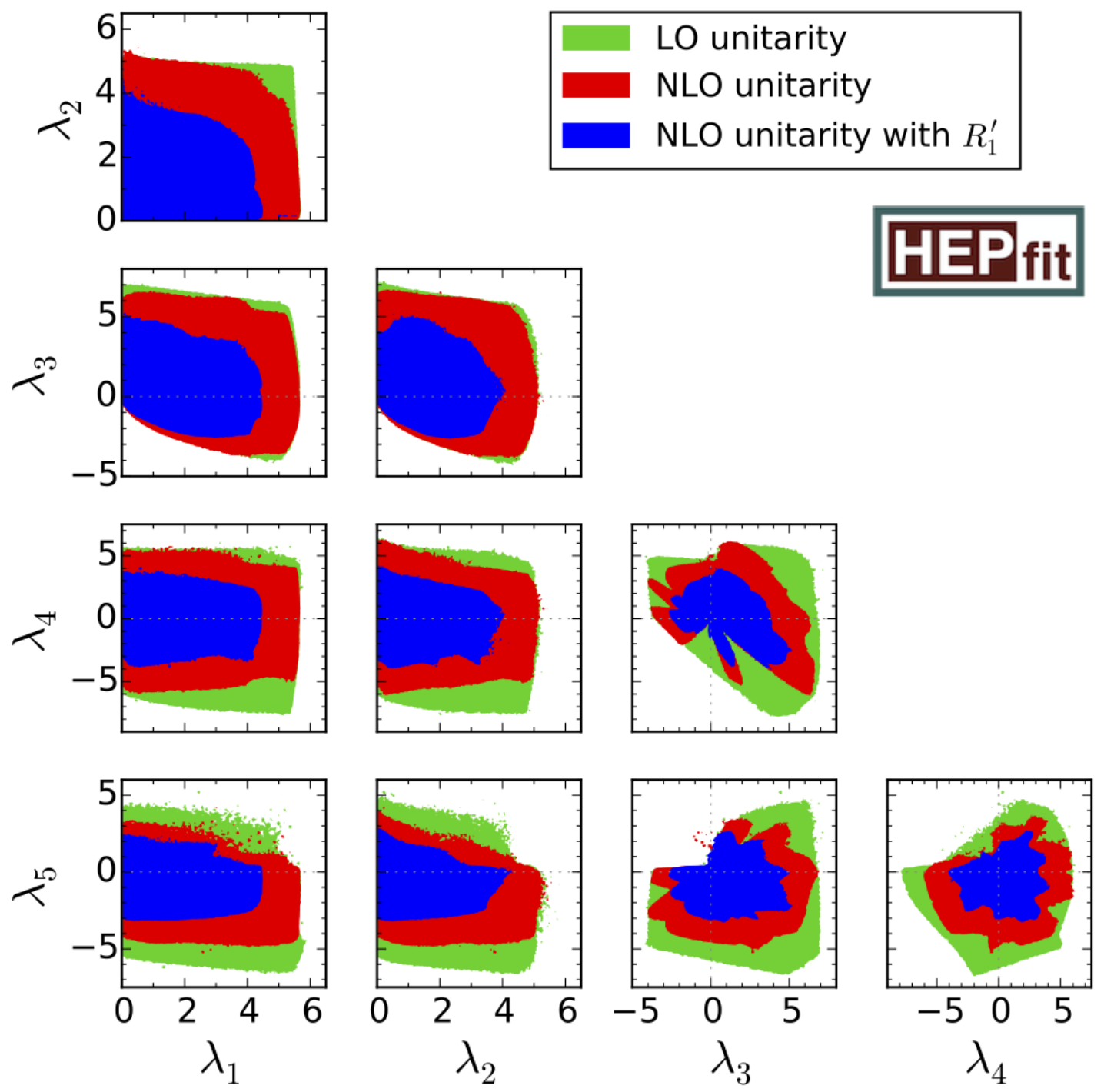

Figure 1. $99.7 \%$ probability regions in the $\lambda_{i}$ vs. $\lambda_{j}$ planes. The green contours show the possible ranges if we impose a stable potential up to $750 \mathrm{GeV}$ and unitarity at LO; the red contours mark the allowed regions if we take the NLO expressions instead, and the blue lines are obtained with the additional assumption that the ratio of NLO and LO unitarity is smaller than one (if the LO value is sufficiently large). The $\lambda_{i}$ values are at the scale $M_{Z}$.

perturbativity requirement $R_{1}^{\prime}<1$, with the first set of constraints being the weakest bound and the last being the strongest bound. While LO unitarity allows for maximal $\left|m_{H}-m_{A}\right|$, $\left|m_{H}-m_{H^{+}}\right|$and $\left|m_{A}-m_{H^{+}}\right|$of $500 \mathrm{GeV}$, the perturbative NLO unitarity conditions sets upper limits on the mass splittings of around $360 \mathrm{GeV} . m_{H^{+}}>m_{H}$ and $m_{A}>m_{H}$ are already almost excluded by LO unitarity for $\lambda_{3}<0$ and $\lambda_{5}>0$, respectively; we can see that after the inclusion of NLO unitarity with the $R_{1}^{\prime}$ condition also other constellations like $\lambda_{3}=0$ feature significantly smaller possible mass differences. 

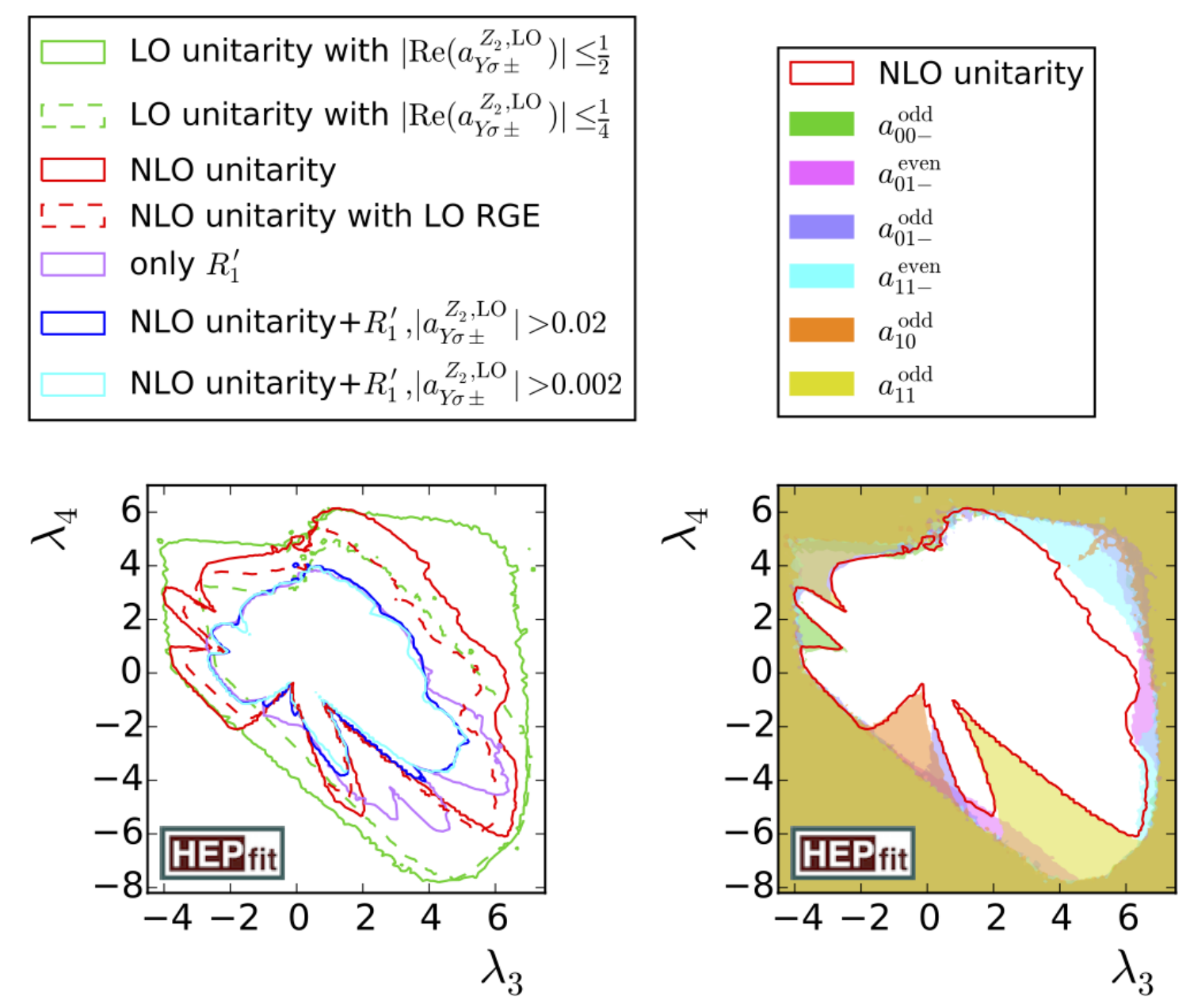

Figure 2. Comparisons of the effects of different constraints in the $\lambda_{4}$ vs. $\lambda_{3}$ plane. Left: the solid green, red, and blue curves have the same meaning here as the shaded regions of the same colour in figure 1. The dashed green curve shows the effect of (arbitrarily) requiring the LO unitarity condition to be more restrictive. The pink curve demonstrates the impact of the perturbativity bounds without the unitarity bounds. The cyan curve requires $\left|a_{Y \sigma \pm}^{\mathbb{Z}_{2}, \mathrm{LO}}\right|>0.002$ in order for the perturbativity bounds to be enforced, rather than 0.02 , leading to no significant change in the allowed parameter space. Right: breakdown of the single effects of the unitarity constraints. Only the most constraining eigenvalues are displayed.

Figure 4 contains the same quartic coupling planes as figure 1, but additionally the experimental data has been taken into account. The blue region survives all theoretical constraints as mentioned at the beginning of section 3.1 and is identical with the blue contours of the previous figures. The unfilled contours have been obtained using only one of the following three sets of inputs in combination with the requirement that the scalar potential is stable up to $750 \mathrm{GeV}$ : the oblique parameters (labelled "STU"); $h$ signal strengths and $H$ and $A$ searches ("Higgs"); $\Delta m_{B_{s}}$ and $\mathcal{B}\left(\bar{B} \rightarrow X_{s} \gamma\right.$ ) ("Flavour"). For the latter two the coloured dashed lines represent type I fits, while the solid lines are the type II contours. In both types, the first set is most constraining for negative $\lambda_{3}$ or 


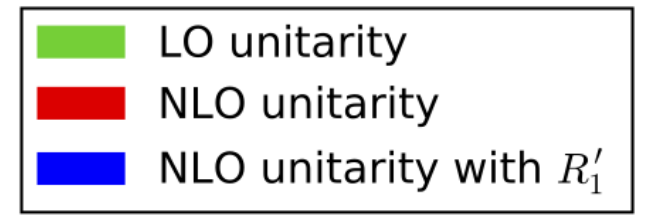

\section{HEP fit $_{\text {fit }}$}
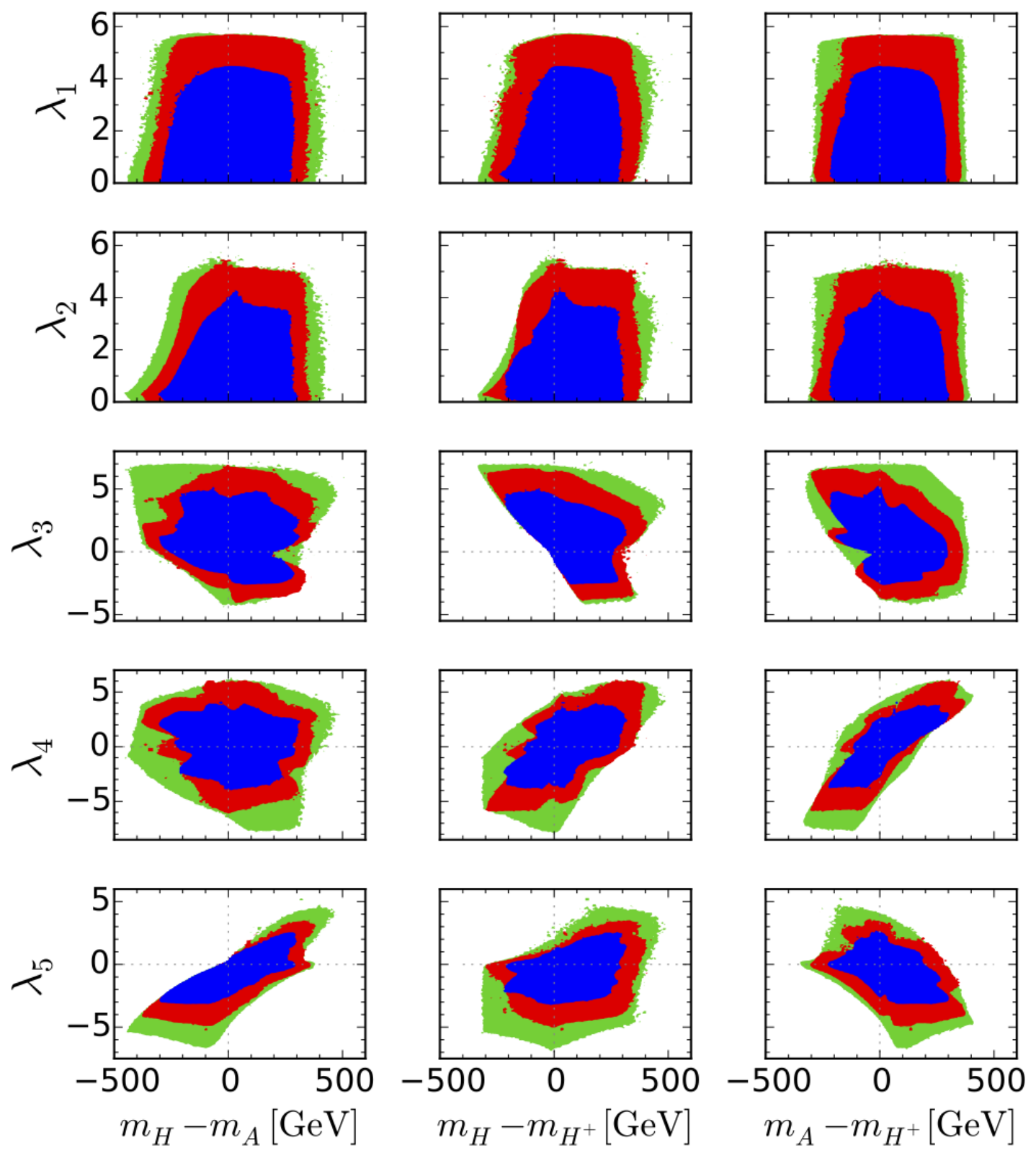

Figure 3. $99.7 \%$ probability regions in the $\lambda_{i}$ vs. $\left(m_{j}-m_{k}\right)$ planes. The colours of the regions have the same meaning as those of figure 1. 

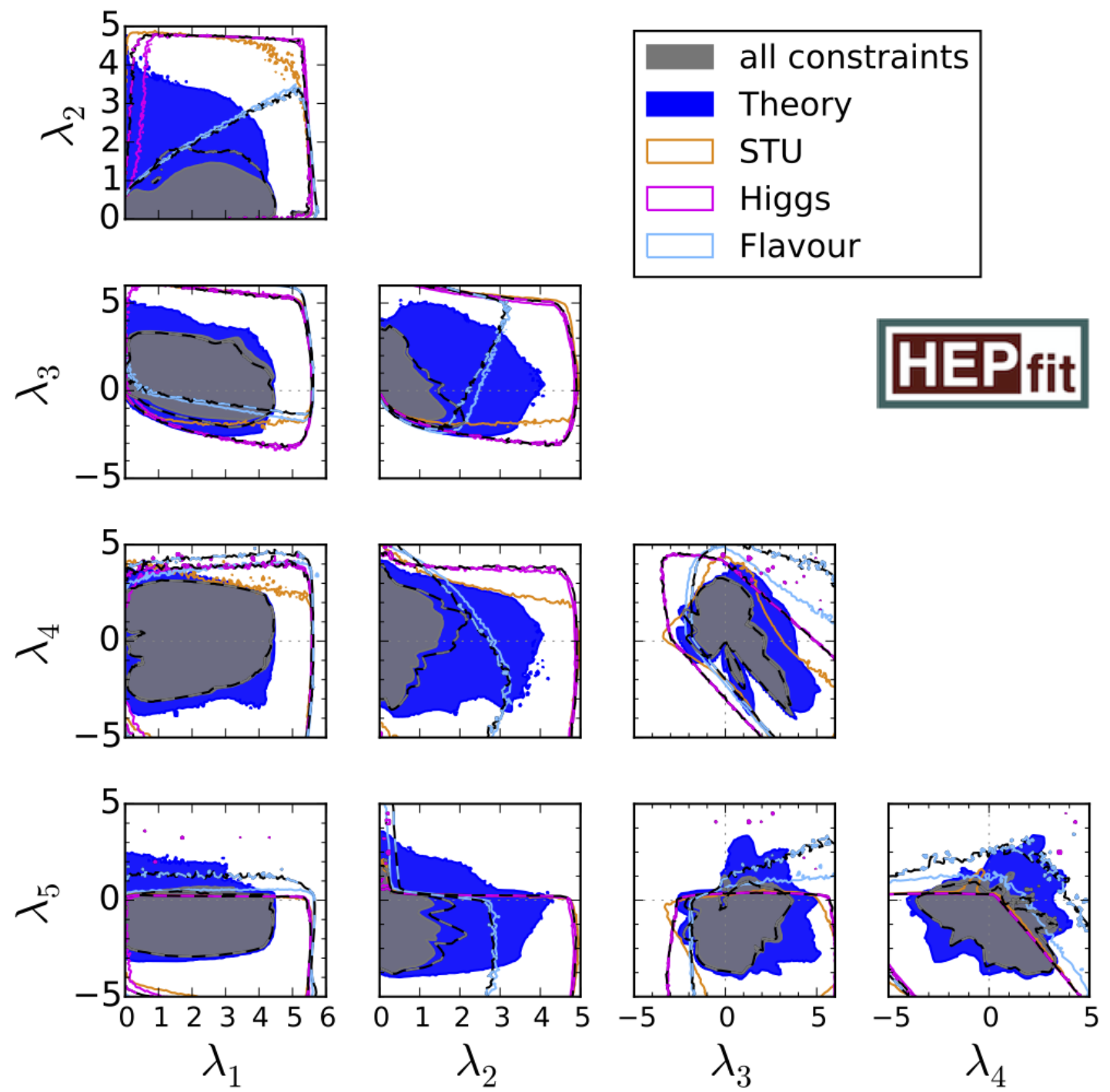

Figure 4. $\lambda_{i}$ vs. $\lambda_{j}$ planes including experimental constraints. The blue shaded regions are the same $99.7 \%$ probability areas as in figure 1, while the orange, pink and light blue lines mark the 95.4\% probability boundaries of fits using only the oblique parameters $(S T U)$, all direct Higgs observables (that is $h$ signal strengths and searches for $H$ and $A$ ) or the flavour observables $\Delta m_{B_{s}}$ and $\mathcal{B}\left(\bar{B} \rightarrow X_{s} \gamma\right)$. The grey contours are compatible with all theoretical and experimental bounds at a probability of $95.4 \%$. The solid lines are understood as the type II contours, the coloured dashed lines represent the corresponding type I fits. 

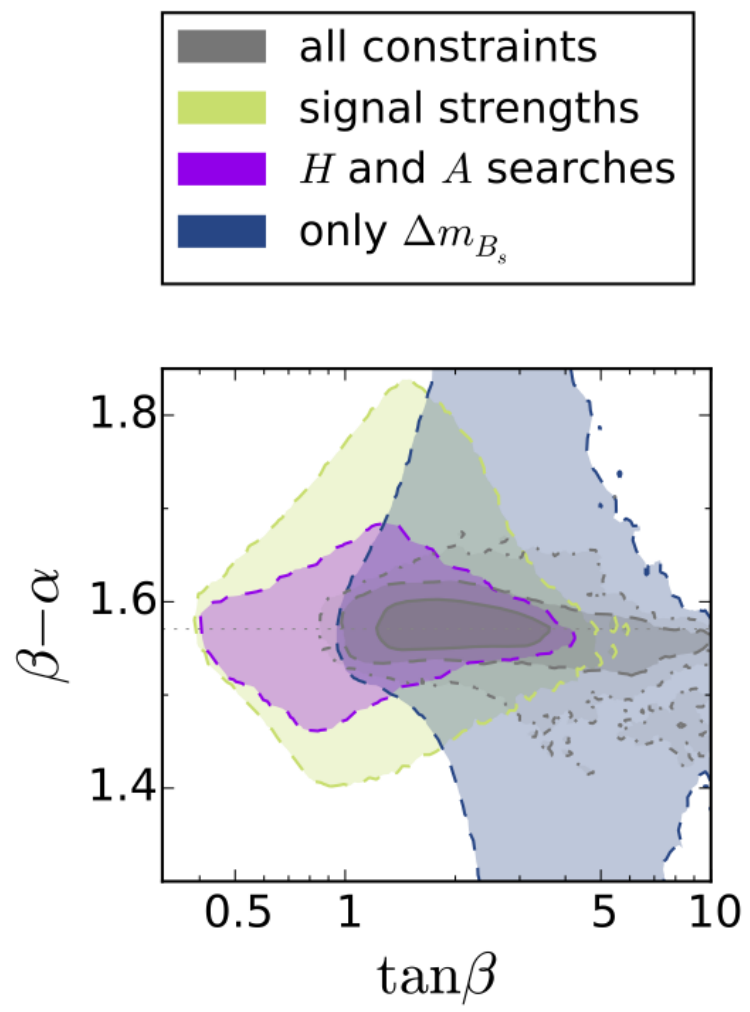

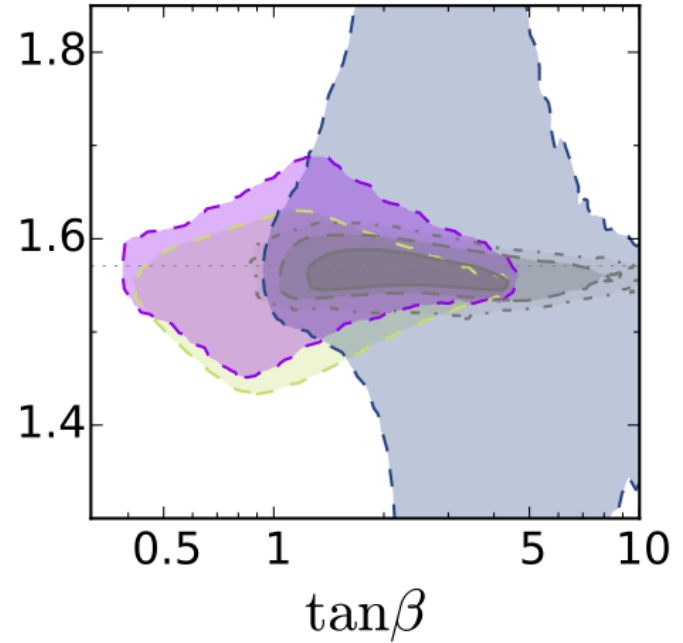

Figure 5. The $\beta-\alpha$ vs. $\tan \beta$ planes of type I (left) and type II (right) with the single contributions of the most important constraints: the $h$ signal strengths in light green, the heavy neutral Higgs searches in pink and the mass difference between the $B_{s}$ and $\bar{B}_{s}$ mesons in dark blue; the grey contours stem from the combined fit to all constraints. The dashed lines represent the $95.4 \%$ probability boundaries, the grey solid and dash-dotted ones the $68.3 \%$ and $99.7 \%$ contours, respectively. The grey dotted line indicates the alignment limit $\beta-\alpha=\pi / 2$.

positive $\lambda_{4}$, the second set excludes $\lambda_{5}>0.4$, and the third set of inputs yields $\lambda_{2}<3.5$ and $\lambda_{3}>-2$. Finally, the grey regions denote the combination of all theoretical and experimental constraints in type II and the grey dashed lines correspond to the type I fits. The allowed $\lambda_{1}$ and $\lambda_{4}$ intervals are similar to the ones obtained in figure 1 , but the other three receive significant additional restrictions from the experimental bounds: with a probability of $95.4 \%, \lambda_{2}$ cannot exceed $1.6(1.2), \lambda_{3}$ has to be within -1.6 and 3.0 $(-1.3$ and 3.1$)$ and the allowed $\lambda_{5}$ interval is between -2.7 and $0.3(-2.7$ and 0.5$)$, when marginalizing over all parameters in type I (type II).

Again turning towards the physical parameters, we show the allowed parameter space in the $\beta-\alpha$ vs. $\tan \beta$ plane for type I and II in the left and right panels of figure 5 , respectively. The most important bounds in this plane comes from the $h$ signal strengths, the heavy Higgs searches and $\Delta m_{B_{s}}$; their $95.4 \%$ bounds are also depicted individually. The Higgs observables strongly constrain the difference between $\alpha$ and $\beta$. In the final fit with all constraints, the deviation from the alignment limit, $|\beta-\alpha-\pi / 2|$, cannot exceed 
0.15 in type I and 0.04 in type II. (This corresponds to a maximal deviation of $\sin (\beta-\alpha)$ from 1 by 0.01 and $7 \cdot 10^{-4}$, respectively.) The mass difference in the $B_{s}$ system sets a type independent lower bound on $\beta$ for the chosen mass priors. More details about the effects of the signal strengths can be found in figures 8 and 9 in appendix A.

In figure 6, we plot the allowed ranges for the heavy Higgs boson masses and their mass differences after imposing the theoretical and experimental constraints for type I and type II. The green, red and blue regions depict the $99.7 \%$ allowed parameter space for the various unitarity conditions discussed above. The orange region is the allowed by the $S T U$ observables at $95.4 \%$. Finally, the grey region is the available parameter space after all the theoretical and experimental constraints are taken into consideration. Even if the perturbative NLO unitarity contour represents a larger probability boundary than the oblique parameter contour ( $99.7 \%$ for the former, $95.4 \%$ for the latter), it is more stringent for masses above $400 \mathrm{GeV}$ and thus the dominant constraint in the high mass regime. It allows for maximal mass splittings between $m_{H}, m_{A}$ and $m_{H^{+}}$of around $100 \mathrm{GeV}$ for masses above $600 \mathrm{GeV}$. After the inclusion of the LHC searches for heavy neutral Higgs bosons, we observe that the remaining parameter space is disconnected. The largest gap occurs around $m_{H, A} \approx 550 \mathrm{GeV}$. The reason for this discontinuity is that our fits are incompatible with the observed ATLAS and CMS diphoton cross sections around this mass. For details, we refer to figures 10 and 11 in appendix A. With a probability of $95.4 \%, H$ and $H^{+}$can be as light as $210 \mathrm{GeV}$, and $m_{A}$ cannot be smaller than approximately $400 \mathrm{GeV}$ in type I. In type II, masses below $600 \mathrm{GeV}$ are excluded at $95.4 \%$ after the inclusion of $\mathcal{B}\left(\bar{B} \rightarrow X_{s} \gamma\right)$ to the fit.

Finally, we address the soft $\mathbb{Z}_{2}$ symmetry breaking parameter $m_{12}^{2}$. In figure 7 we show its dependence on $\tan \beta$ and the $H$ Higgs mass in the two discussed types. While for the theoretical set of constraints a strong correlation between the heavy Higgs mass and $m_{12}^{2}$ is visible, this gets somewhat relaxed if one adds experimental data to the fit. This is due to the flavour constraints, which favour larger $\tan \beta$ and $m_{H^{+}}$values. The most important result here is that an unbroken $\mathbb{Z}_{2}$ symmetry can be excluded with a probability of $95.4 \%$ in the combined fit to the type II; the single sets of constraints are individually compatible with an exact $\mathbb{Z}_{2}$ symmetry. The lowest $95.4 \%$ allowed value for $m_{12}^{2}$ is $(370 \mathrm{GeV})^{2}$, if we marginalize over all other parameters.

\section{Conclusions}

The determination of the NLO unitarity constraints to the 2HDM with a softly broken $\mathbb{Z}_{2}$ symmetry mitigates the problem of how to tame higher order contributions involving large quartic couplings. The expressions have been derived in ref. [57], and in this article we perform the first general fits to them in the $2 \mathrm{HDM}$ of type I and II, making use of the publicly available package HEPfit. One important result is that wavefunction renormalization contributions can be safely neglected in these models. In our fits we also apply the suppression of non-perturbative higher order contributions with the $R_{1}^{\prime}$ condition, requiring that the NLO part cannot be larger in magnitude than the LO contribution if the latter is not accidentally small. We find that both steps, going from LO to NLO unitarity and comparing NLO unitarity with $R_{1}^{\prime}$-perturbative NLO unitarity, individually put strong 


\begin{tabular}{|l|}
\hline LO unitarity \\
NLO unitarity \\
NLO unitarity with $R_{1}^{\prime}$ \\
STU \\
all constraints
\end{tabular}
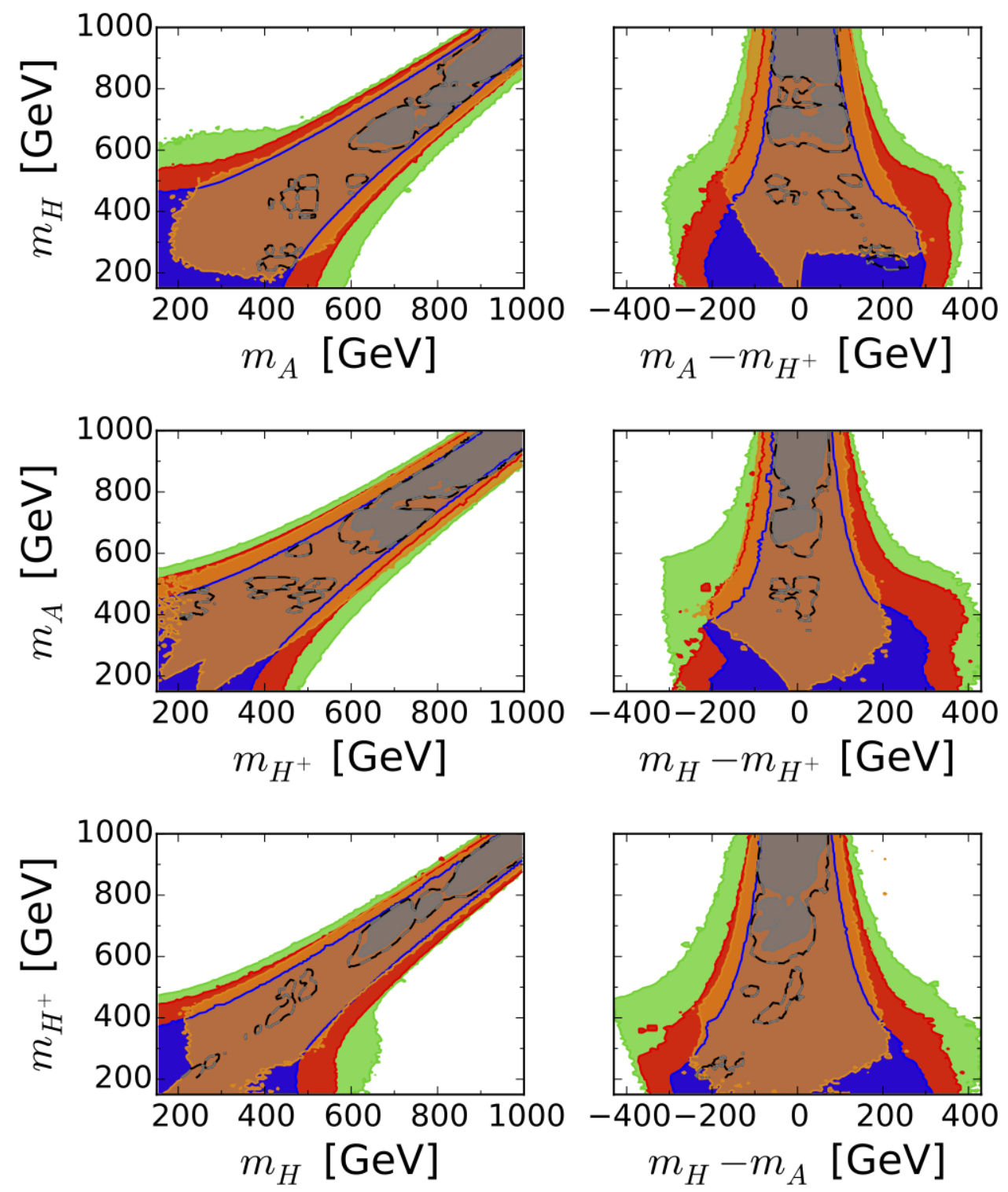

Figure 6. Allowed regions in the heavy Higgs boson masses and their mass differences planes in the 2HDM of type I (dashed lines) and type II (solid lines). The unitarity bounds to the green, red and blue regions are meant at a probability of $99.7 \%$, and the orange and grey lines mark the $95.4 \%$ boundaries. 

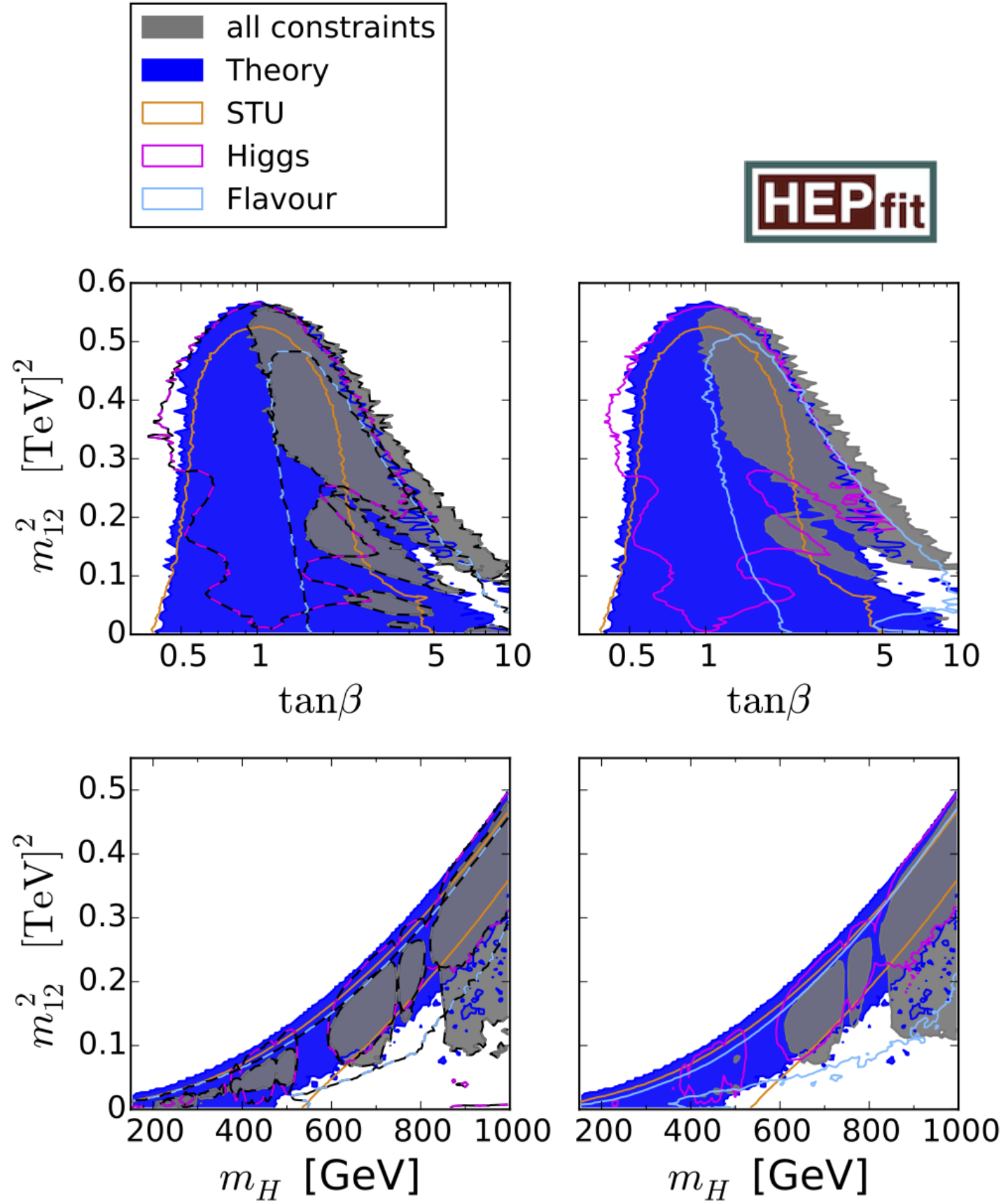

Figure 7. $m_{12}^{2}$ vs. $\tan \beta$ and $m_{12}^{2}$ vs. $m_{H}$ planes in type I (left) and type II (right). The colours have the same meaning than in figure 4 with the difference that also the grey type I contour was filled here. 
bounds on the $2 \mathrm{HDM}$ parameters. If we add all other relevant theoretical constraints, that is stability and positivity of the scalar potential up to a scale of $750 \mathrm{GeV}$, the quartic $\lambda_{i}$ couplings cannot exceed 5.8 in magnitude and the mass differences between $m_{H}, m_{A}$ and $m_{H^{+}}$cannot be larger than approximately $360 \mathrm{GeV}$. (The latter even reduces to maximally $100 \mathrm{GeV}$ for heavy Higgs masses above $800 \mathrm{GeV}$.) To our knowledge, this currently represents the strongest reliable bound on the mass splittings.

Moreover, we have added the most relevant experimental constraints to the fit: the electroweak precision data in form of the oblique parameters, the complete set of LHC Run I results and the most important flavour observables. These bounds constrain the quartic couplings even further: the allowed intervals for the quartic couplings are

$$
0 \leq \lambda_{1}<4.2, \quad 0 \leq \lambda_{2}<1.6, \quad-1.6<\lambda_{3}<3.0, \quad-2.5<\lambda_{4}<2.9, \quad-2.7<\lambda_{5}<0.3
$$

in type I and

$$
0 \leq \lambda_{1}<4.2, \quad 0 \leq \lambda_{2}<1.2, \quad-1.3<\lambda_{3}<3.1, \quad-2.5<\lambda_{4}<2.9, \quad-2.7<\lambda_{5}<0.5
$$

in type II with a probability of $95.4 \%$. For the physical parameters, we find that $\tan \beta$ cannot be smaller than 1 in both discussed types of the 2HDM. The deviation from the alignment limit $|\beta-\alpha-\pi / 2|$ cannot exceed 0.15 (0.04) in type I (type II). In type I the global fit produces lower $95.4 \%$ bounds of $210 \mathrm{GeV}$ for $m_{H}$ and $m_{H^{+}}$and $410 \mathrm{GeV}$ for $m_{A}$, while these limits are around $650 \mathrm{GeV}$ for all three heavy Higgs masses in type II. In the latter case, also an unbroken $\mathbb{Z}_{2}$ symmetry can be ruled out at $95.4 \%$; the soft $\mathbb{Z}_{2}$ breaking parameter $m_{12}^{2}$ has to be larger than $(370 \mathrm{GeV})^{2}$.

\section{Acknowledgments}

We thank Marco Fedele, Enrico Franco, Benjamín Grinstein, Ayan Paul, Maurizio Pierini, Luca Silvestrini, and Patipan Uttayarat for useful discussions. The research leading to these results has received funding from the European Research Council under the European Union's Seventh Framework Programme (FP/2007-2013) / ERC Grant Agreement n. 279972. This work was supported in part by the MIUR-FIRB under grant no. RBFR12H1MW.

\section{A Additional figures}

In this appendix, we present supplementary figures of the 2HDM parameter space: dedicated fits of the different signal strength measurements and $H$ and $A$ searches in type I and II in figures 8 to 11, and the quartic couplings of the so-called Higgs basis in figures 12 and 13 .

In figure 8 , we show the effect of the $h$ signal strengths on the $\beta-\alpha$ vs. $\tan \beta$ plane for $2 \mathrm{HDM}$ of type I. In the top left panel of figure 8 , the effect of considering all the five signal strengths in the "ggF +tth" production modes on this plane is represented by the orange shaded region, considering all the five signal strengths in the "VBF+VH" production 

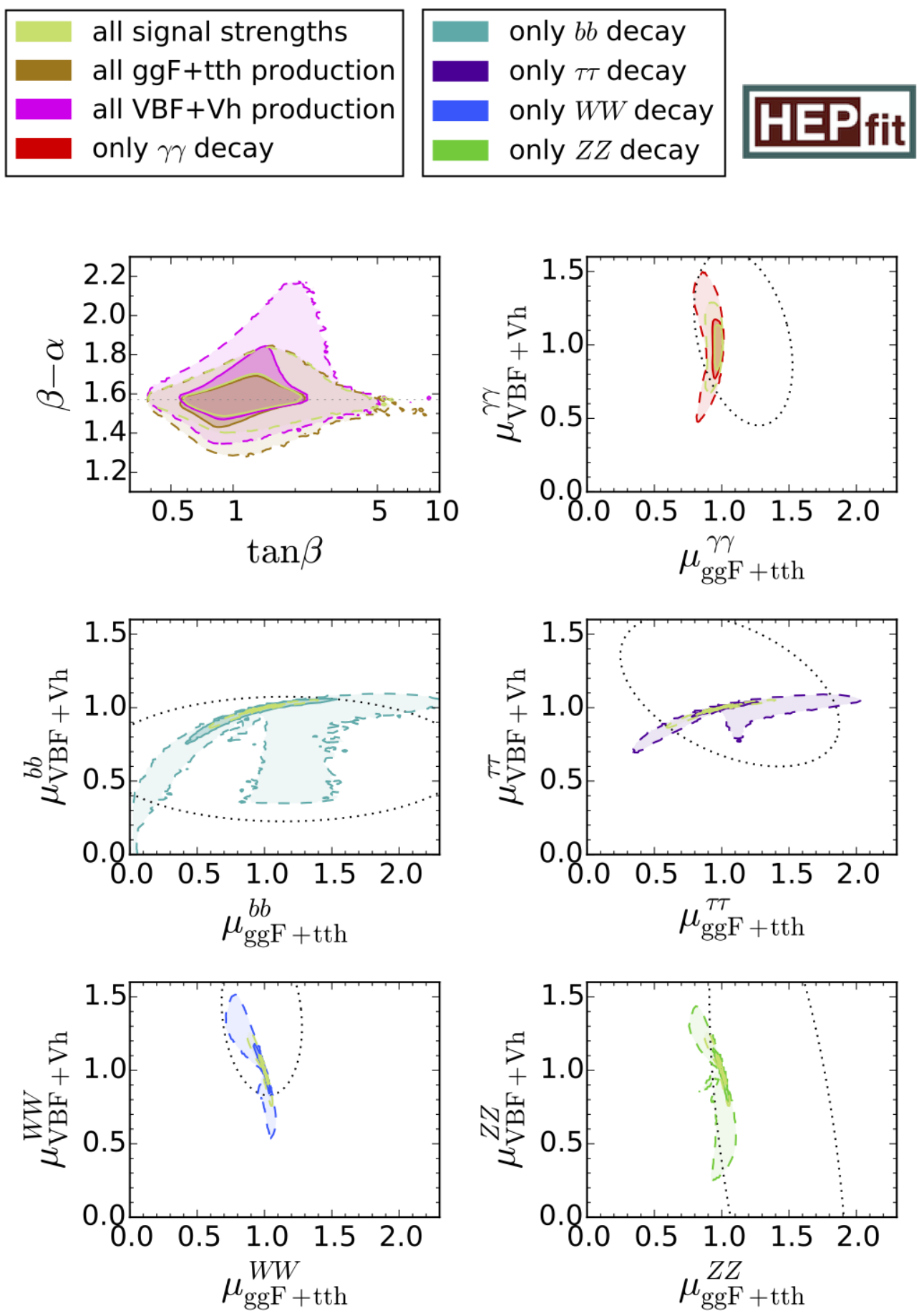

Figure 8. $68.3 \%$ (solid) and $95.4 \%$ (dashed) regions in the $\beta-\alpha$ vs. $\tan \beta$ plane and different signal strengths in type I. The colours of the single decay channels match the ones chosen for the official combination of ATLAS and CMS signal strengths [17], which are also approximated by the black dotted ellipses. In the top left panel, we also mark the alignment limit $\beta-\alpha=\pi / 2$ by a grey dotted line. 
modes are shown in the pink region, and the light green shaded region depicts the allowed parameter space when all the ten signal strengths are taken into consideration. In order to compare the latter with the effect of each of the five decay modes individually, we separately plot the single decay modes at a time on the rest of the panels of figure 8 overlaid with the fit with all signal strengths. For each of these additional panels we also indicate the latest $8 \mathrm{TeV}$ signal strength correlation contours at $68 \% \mathrm{CL}$ taken from ref. [17]. In all the panels, the filled regions with solid (dashed) lines represent the $68.3 \%$ (95.4\%) probability contours as obtained from the fits. Figure 9 displays the same panels as figure 8 but for type II.

Figures 10 and 11 compare the most important constraints on the heavy Higgs masses vs. $\tan \beta$ planes (left column) and on the heavy Higgs masses vs. $\beta-\alpha$ planes (right column) in type I and type II, respectively. In the first four panels, the relevant $H$ (top row) and $A$ (second row) searches are represented by the shaded regions, which they exclude. For the attribution of the colours, we refer to the legends. The left panel of the bottom row of figures 10 and 11 shows in beige the constraint from the mass difference in the $B_{s}$ system on the charged Higgs mass vs. $\tan \beta$ plane, which excludes $\tan \beta<1$ for the chosen $m_{H^{+}}$range. Figure 11 additionally features the constraint from $\mathcal{B}\left(\bar{B} \rightarrow X_{s} \gamma\right)$ disfavouring charged Higgs masses below $410 \mathrm{GeV}$. The grey contours in all the panels of figures 10 and 11 depict the allowed parameter space after all of the theoretical and experimental constraint have been taken into account. All contours represent the $95.4 \%$ probability boundaries. In the $m_{H}$ and $m_{A}$ planes, the searches for neutral Higgs particles mainly disfavour very small and very large values of $\tan \beta$. Around $550 \mathrm{GeV}$ even all $\tan \beta$ values are incompatible with the measured diphoton events at ATLAS and CMS. In the data of both collaborations, the observed upper limits on $\sigma \mathcal{B}$ are significantly larger than the expected exclusion limits at this invariant mass; an excess which cannot be explained in the context of a 2HDM of type I or II. The grey contours reflect the features of all important constraints as well as their interplay: also in the $m_{H / A}$ vs. $\tan \beta$ planes, small $\tan \beta$ values are excluded in the fit with all constraints. Light $m_{H}$ and $m_{A}$ are excluded in type II (figure 11) because the masses of the neutral Higgs bosons cannot be very different from the $H^{+}$mass due to unitarity (see figure 6 ).

Instead of the general parametrisation of the potential in (2.1), one is free to choose a basis in which only one of the two transformed doublets, $H_{1}$ and $H_{2}$, obtain a VEV. This basis is called the Higgs basis [82, 83], and its potential can be written as

$$
\begin{aligned}
V= & Y_{1} H_{1}^{\dagger} H_{1}+Y_{2} H_{2}^{\dagger} H_{2}+Y_{3}\left(H_{1}^{\dagger} H_{2}+H_{2}^{\dagger} H_{1}\right)+\frac{1}{2} Z_{1}\left(H_{1}^{\dagger} H_{1}\right)^{2}+\frac{1}{2} Z_{2}\left(H_{2}^{\dagger} H_{2}\right)^{2} \\
& +Z_{3}\left(H_{1}^{\dagger} H_{1}\right)\left(H_{2}^{\dagger} H_{2}\right)+Z_{4}\left(H_{1}^{\dagger} H_{2}\right)\left(H_{2}^{\dagger} H_{1}\right)+\frac{1}{2} Z_{5}\left[\left(H_{1}^{\dagger} H_{2}\right)^{2}+\left(H_{2}^{\dagger} H_{1}\right)^{2}\right] \\
& +\left[Z_{6}\left(H_{1}^{\dagger} H_{1}\right)+Z_{7}\left(H_{2}^{\dagger} H_{2}\right)\right]\left(H_{1}^{\dagger} H_{2}+H_{2}^{\dagger} H_{1}\right) .
\end{aligned}
$$

Only five of the seven quartic couplings $Z_{i}$ are linearly independent. One can see from figure 12 that they get constrained by the different unitarity conditions in a similar way than the $\lambda_{i}$ in figure 1 , with the $R_{1}^{\prime}$-perturbative NLO expressions being stronger than simple NLO unitarity, which itself is an improvement of LO unitarity. While the latter does not allow for $\left|Z_{i}\right|>9$, NLO unitarity (with $R_{1}^{\prime}$ ) sets upper limits of approximately 8 (5) on the absolute values of the $Z_{i}$. Analogous to figure 4 , we also show the impact of 

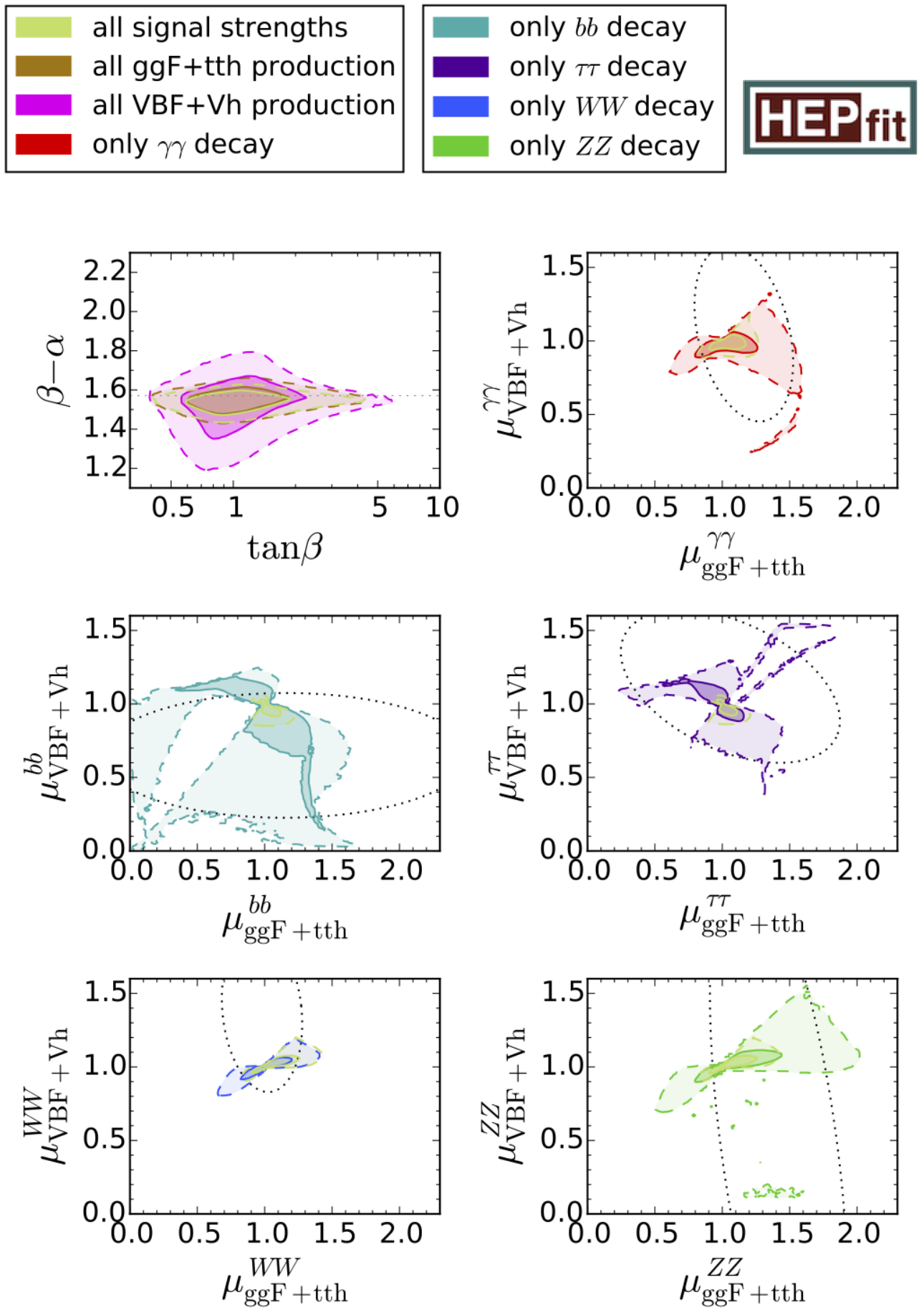

Figure 9. Same as figure 8 but for type II. 

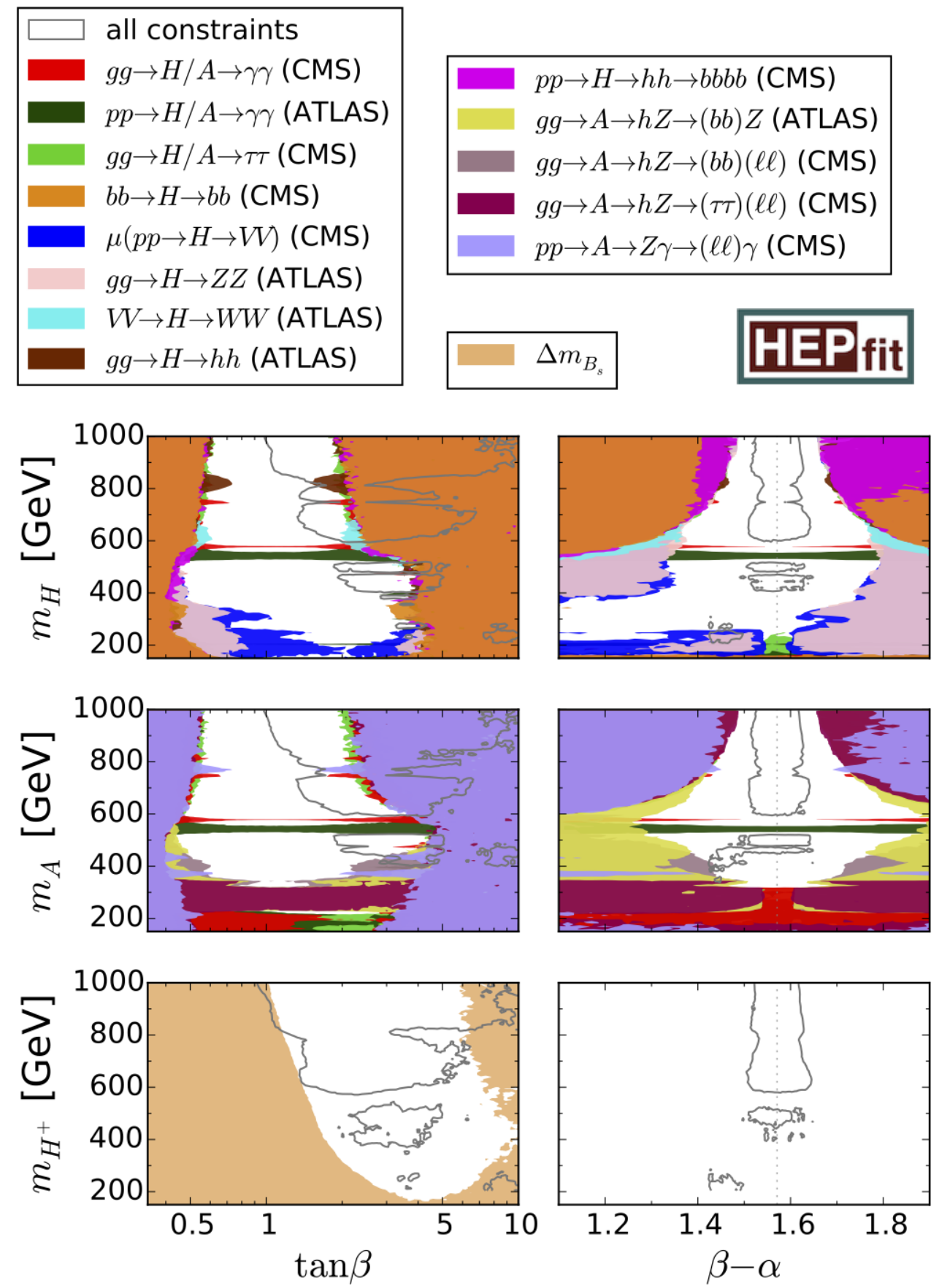

Figure 10. Exclusion boundaries for type I fits in the heavy Higgs mass vs. $\tan \beta$ (left column) and heavy Higgs mass vs. $\beta-\alpha$ (right column) at $95.4 \%$ probability. The grey contour denotes the available parameter space at $95.4 \%$ probability, after imposing all the theoretical and experimental constraints. 

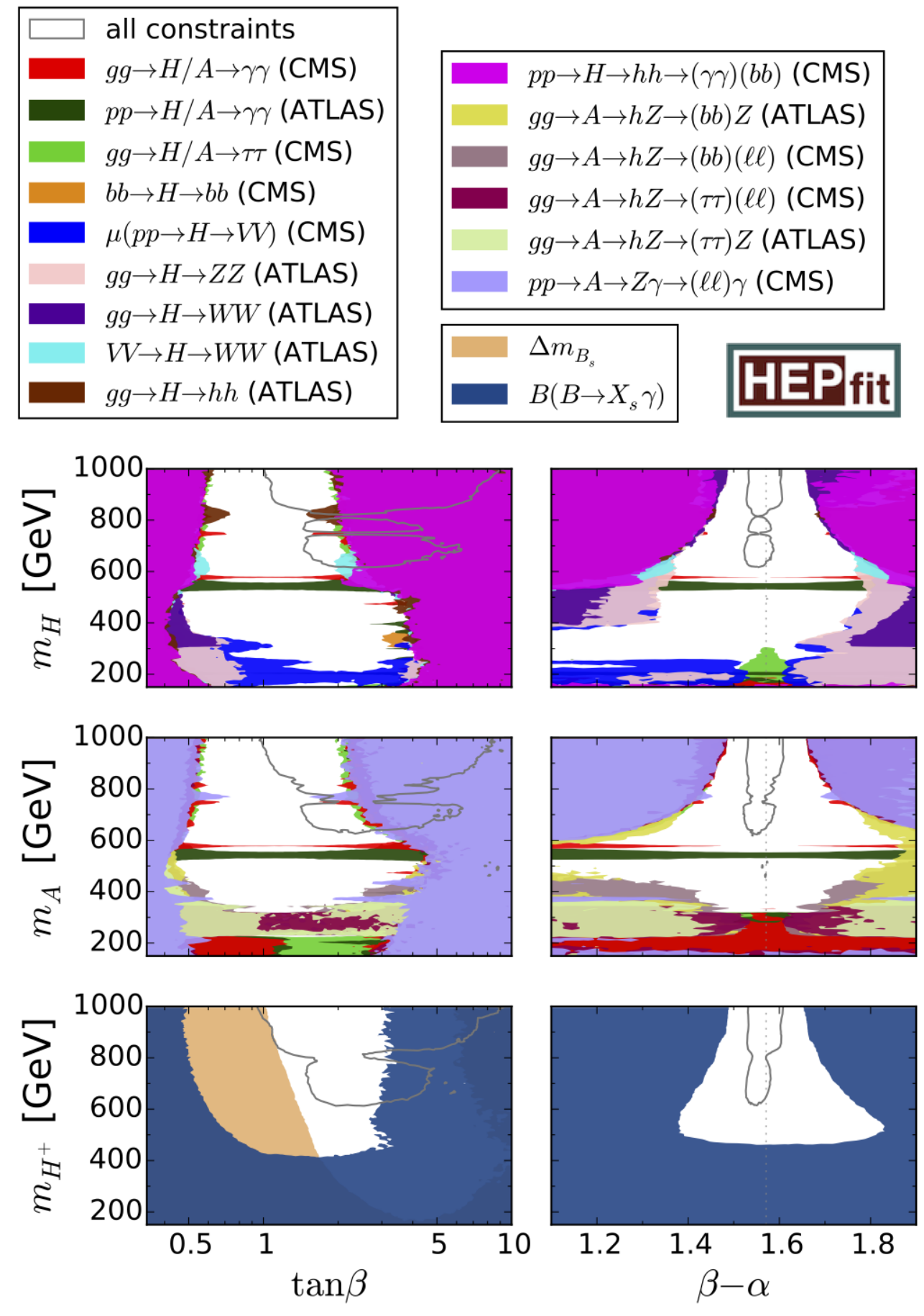

Figure 11. Like figure 10 but for type II fits. 

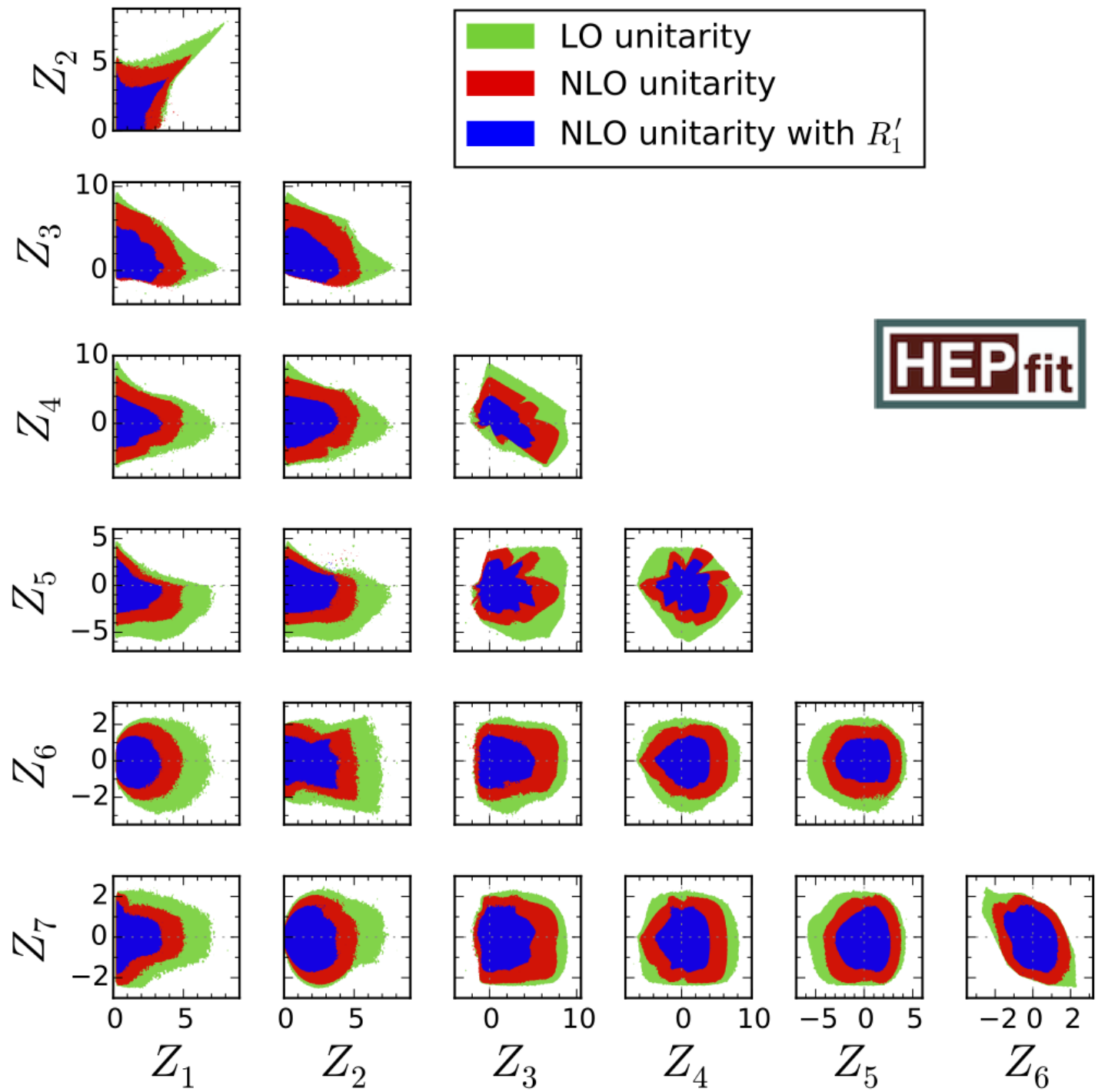

Figure 12. Planes of the quartic couplings $Z_{i}$ of the Higgs basis parametrisation from (A.1). The colours are analogous to figure 1.

the experimental constraints on the $Z_{i}$ vs. $Z_{j}$ planes in figure 13 . Especially $Z_{1}, Z_{6}$ and $Z_{7}$ suffer strong additional restrictions from the experiments.

\section{B Diagonal entries of the NLO $S$-matrix}

For the reader's convenience we list the minimal set of elements of the matrix $\mathbf{a}_{0}$ needed to write its eigenvalues, $a_{0}$, at next-to-leading order accuracy in the limit that the wavefunction renormalization contribution is neglected. In what follows, each $B_{N}$ corresponds in this approximation to eq. (B.N) of appendix B of ref. [57]. The complete expressions for 

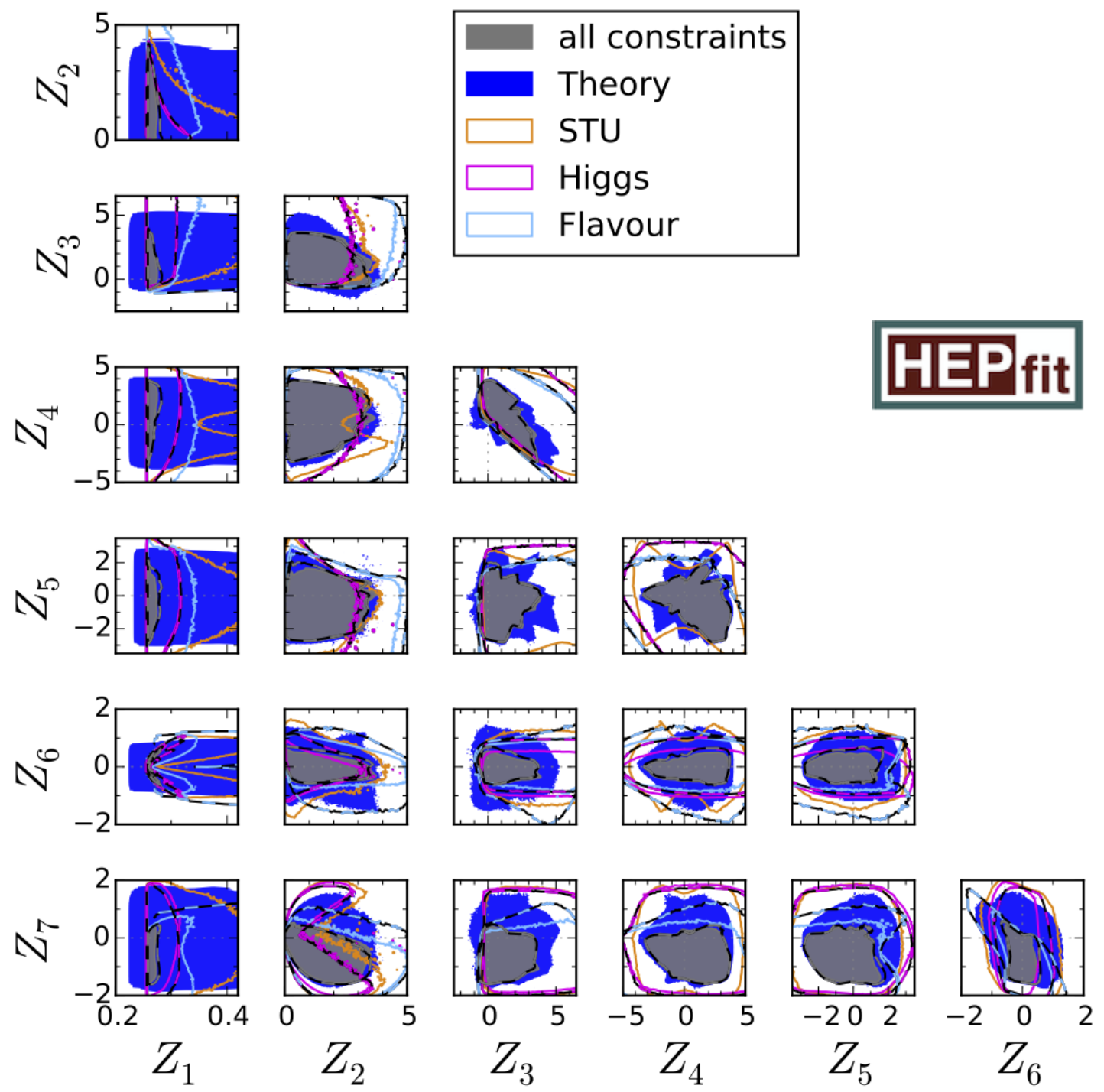

Figure 13. Planes of the quartic couplings $Z_{i}$ of the Higgs basis parametrisation from (A.1) including experimental inputs. The colours are analogous to figure 4.

$\mathbf{a}_{0}$ can be found in appendices $\mathrm{B}$ and $\mathrm{C}$ of ref. [57].

$$
\begin{aligned}
& B_{1}=-3 \lambda_{1}+\frac{9}{2} \beta_{\lambda_{1}}+\frac{1}{16 \pi^{2}}(i \pi-1)\left(9 \lambda_{1}^{2}+\left(2 \lambda_{3}+\lambda_{4}\right)^{2}\right) \\
& B_{2}=-3 \lambda_{2}+\frac{9}{2} \beta_{\lambda_{2}}+\frac{1}{16 \pi^{2}}(i \pi-1)\left(9 \lambda_{2}^{2}+\left(2 \lambda_{3}+\lambda_{4}\right)^{2}\right) \\
& B_{3}=-\left(2 \lambda_{3}+\lambda_{4}\right)+\frac{3}{2}\left(2 \beta_{\lambda_{3}}+\beta_{\lambda_{4}}\right)+\frac{3}{16 \pi^{2}}(i \pi-1)\left(\lambda_{1}+\lambda_{2}\right)\left(2 \lambda_{3}+\lambda_{4}\right) \\
& B_{4}=-\left(\lambda_{3}+2 \lambda_{4}\right)+\frac{3}{2}\left(\beta_{\lambda_{3}}+2 \beta_{\lambda_{4}}\right)+\frac{1}{16 \pi^{2}}(i \pi-1)\left(\lambda_{3}^{2}+4 \lambda_{3} \lambda_{4}+4 \lambda_{4}^{2}+9 \lambda_{5}^{2}\right) \\
& B_{6}=-3 \lambda_{5}+\frac{9}{2} \beta_{\lambda_{5}}+\frac{6}{16 \pi^{2}}(i \pi-1)\left(\lambda_{3}+2 \lambda_{4}\right) \lambda_{5}
\end{aligned}
$$




$$
\begin{aligned}
B_{7} & =-\lambda_{1}+\frac{3}{2} \beta_{\lambda_{1}}+\frac{1}{16 \pi^{2}}(i \pi-1)\left(\lambda_{1}^{2}+\lambda_{4}^{2}\right) \\
B_{8} & =-\lambda_{2}+\frac{3}{2} \beta_{\lambda_{2}}+\frac{1}{16 \pi^{2}}(i \pi-1)\left(\lambda_{2}^{2}+\lambda_{4}^{2}\right) \\
B_{9} & =-\lambda_{4}+\frac{3}{2} \beta_{\lambda_{4}}+\frac{1}{16 \pi^{2}}(i \pi-1)\left(\lambda_{1}+\lambda_{2}\right) \lambda_{4} \\
B_{13} & =-\lambda_{3}+\frac{3}{2} \beta_{\lambda_{3}}+\frac{1}{16 \pi^{2}}(i \pi-1)\left(\lambda_{3}^{2}+\lambda_{5}^{2}\right) \\
B_{15} & =-\lambda_{5}+\frac{3}{2} \beta_{\lambda_{5}}+\frac{2}{16 \pi^{2}}(i \pi-1) \lambda_{3} \lambda_{5} \\
B_{19} & =-\left(\lambda_{3}-\lambda_{4}\right)+\frac{3}{2}\left(\beta_{\lambda_{3}}-\beta_{\lambda_{4}}\right)+\frac{1}{16 \pi^{2}}(i \pi-1)\left(\lambda_{3}-\lambda_{4}\right)^{2} \\
B_{20} & =-\lambda_{1}+\frac{3}{2} \beta_{\lambda_{1}}+\frac{1}{16 \pi^{2}}(i \pi-1)\left(\lambda_{1}^{2}+\lambda_{5}^{2}\right) \\
B_{21} & =-\lambda_{2}+\frac{3}{2} \beta_{\lambda_{2}}+\frac{1}{16 \pi^{2}}(i \pi-1)\left(\lambda_{2}^{2}+\lambda_{5}^{2}\right) \\
B_{22} & =-\lambda_{5}+\frac{3}{2} \beta_{\lambda_{5}}+\frac{1}{16 \pi^{2}}(i \pi-1)\left(\lambda_{1}+\lambda_{2}\right) \lambda_{5} \\
B_{30} & =-\left(\lambda_{3}+\lambda_{4}\right)+\frac{3}{2}\left(\beta_{\lambda_{3}}+\beta_{\lambda_{4}}\right)+\frac{1}{16 \pi^{2}}(i \pi-1)\left(\lambda_{3}+\lambda_{4}\right)^{2}
\end{aligned}
$$

For completeness, the leading terms of the beta functions appearing in the above equations are

$$
\begin{aligned}
& 16 \pi^{2} \beta_{\lambda_{1}}=12 \lambda_{1}^{2}+4 \lambda_{3}^{2}+4 \lambda_{3} \lambda_{4}+2 \lambda_{4}^{2}+2 \lambda_{5}^{2}, \\
& 16 \pi^{2} \beta_{\lambda_{2}}=12 \lambda_{2}^{2}+4 \lambda_{3}^{2}+4 \lambda_{3} \lambda_{4}+2 \lambda_{4}^{2}+2 \lambda_{5}^{2}, \\
& 16 \pi^{2} \beta_{\lambda_{3}}=4 \lambda_{3}^{2}+2 \lambda_{4}^{2}+\left(\lambda_{1}+\lambda_{2}\right)\left(6 \lambda_{3}+2 \lambda_{4}\right)+2 \lambda_{5}^{2}, \\
& 16 \pi^{2} \beta_{\lambda_{4}}=\left(2 \lambda_{1}+2 \lambda_{2}+8 \lambda_{3}\right) \lambda_{4}+4 \lambda_{4}^{2}+8 \lambda_{5}^{2}, \\
& 16 \pi^{2} \beta_{\lambda_{5}}=\left(2 \lambda_{1}+2 \lambda_{2}+8 \lambda_{3}+12 \lambda_{4}\right) \lambda_{5} .
\end{aligned}
$$

It is worth mentioning that here only the $\mathrm{LO}$ expressions for the $\beta$ functions should be used in order to be consistent with the order of perturbation theory. For the running in the fits we apply NLO RGE.

\section{Experimental inputs}

In the tables 2 to 5 we list all used experimental inputs for our fits with their corresponding references. 


\begin{tabular}{|c|c|ccc|}
\hline Pseudo-observable & Value & \multicolumn{3}{|c|}{ Correlation matrix } \\
\hline$S$ & $0.09 \pm 0.10$ & 1 & 0.86 & -0.54 \\
$T$ & $0.10 \pm 0.12$ & 0.86 & 1 & -0.81 \\
$U$ & $0.01 \pm 0.09$ & -0.54 & -0.81 & 1 \\
\hline
\end{tabular}

Table 2. S, T, and $U$ values and correlations from [71].

\begin{tabular}{|c|c|cc|}
\hline Signal strength & Value & Correlation matrix \\
\hline$\mu_{\mathrm{ggF}+\mathrm{tth}}^{\gamma \gamma}$ & $1.16 \pm 0.26$ & 1 & -0.30 \\
$\mu_{\mathrm{VBF}+\mathrm{Vh}}^{\gamma \gamma}$ & $1.05 \pm 0.43$ & -0.30 & 1 \\
\hline$\mu_{\mathrm{ggF}+\mathrm{tth}}^{b b}$ & $1.15 \pm 0.97$ & 1 & $4.5 \cdot 10^{-3}$ \\
$\mu_{\mathrm{VBF}+\mathrm{Vh}}^{b b}$ & $0.65 \pm 0.30$ & $4.5 \cdot 10^{-3}$ & 1 \\
\hline$\mu_{\mathrm{ggF}+\mathrm{tth}}^{\tau \tau}$ & $1.06 \pm 0.58$ & 1 & -0.43 \\
$\mu_{\mathrm{VBF}+\mathrm{Vh}}^{\tau \tau}$ & $1.12 \pm 0.36$ & -0.43 & 1 \\
\hline$\mu_{\mathrm{ggF}+\mathrm{tth}}^{W W}$ & $0.98 \pm 0.21$ & 1 & -0.14 \\
$\mu_{\mathrm{VBF}+\mathrm{Vh}}^{W W}$ & $1.38 \pm 0.39$ & -0.14 & 1 \\
\hline$\mu_{\mathrm{ggF}+\mathrm{tth}}^{Z Z}$ & $1.42 \pm 0.35$ & 1 & -0.49 \\
$\mu_{\mathrm{VBF}+\mathrm{Vh}}^{Z Z}$ & $0.47 \pm 1.37$ & -0.49 & 1 \\
\hline
\end{tabular}

Table 3. $h$ signal strengths from figure 13 and table 14 of [17]. 


\begin{tabular}{|c|c|c|c|}
\hline Channel & Experiment & Source & Mass range $(\mathrm{GeV})$ \\
\hline \multirow{2}{*}{$g g \rightarrow H / A \rightarrow \tau \tau$} & ATLAS & Figure 11a of [84] & $90-1000$ \\
\hline & CMS & Figure 10 (left) of [85] & $90-1000$ \\
\hline \multirow{2}{*}{$b \bar{b} \rightarrow H / A \rightarrow \tau \tau$} & ATLAS & Figure $11 \mathrm{~b}$ of $[84]$ & $90-1000$ \\
\hline & $\mathrm{CMS}$ & Figure 10 (right) of [85] & $90-1000$ \\
\hline \multirow{2}{*}{$g g \rightarrow H / A \rightarrow \gamma \gamma$} & ATLAS & Figure $4,[86]$ & $65-600$ \\
\hline & CMS & Figure 7 (left) of [87] & $150-850$ \\
\hline$b \bar{b} \rightarrow H / A \rightarrow b \bar{b}$ & CMS & Figure 6 of [88] & $100-900$ \\
\hline$g g \rightarrow H \rightarrow W W$ & ATLAS & Figure 13 (left) of [89] & $300-1500$ \\
\hline$W W / Z Z \rightarrow H \rightarrow W W$ & ATLAS & Figure 13 (right) of [89] & $300-1500$ \\
\hline$g g \rightarrow H \rightarrow Z Z$ & ATLAS & Figure 12a of [90] & $140-1000$ \\
\hline$W W / Z Z \rightarrow H \rightarrow Z Z$ & ATLAS & Figure $12 \mathrm{~b}$ of $[90]$ & $140-1000$ \\
\hline$p p \rightarrow H \rightarrow Z Z^{3}$ & CMS & Figure 7 (bottom right) of [91] & $150-1000$ \\
\hline$g g \rightarrow H \rightarrow h h$ & ATLAS & Figure 6 of [92] & $260-1000$ \\
\hline$p p \rightarrow H \rightarrow h h[\rightarrow(b \bar{b})(\tau \tau)]$ & CMS & Figure $5 \mathrm{a}$ of [93] & $300-1000$ \\
\hline$p p \rightarrow H \rightarrow h h \rightarrow(\gamma \gamma)(b \bar{b})$ & CMS & Figure 8 of [94] & $250-1100$ \\
\hline$p p \rightarrow H \rightarrow h h \rightarrow(b \bar{b})(b \bar{b})$ & CMS & Figure 5 (left) of [95] & $270-1100$ \\
\hline$g g \rightarrow A \rightarrow h Z \rightarrow(\tau \tau)(\ell \ell)$ & CMS & Figure 10 (left) of [96] & $220-350$ \\
\hline$g g \rightarrow A \rightarrow h Z \rightarrow(b \bar{b})(\ell \ell)$ & CMS & Figure 3 of [97] & $225-600$ \\
\hline$g g \rightarrow A \rightarrow h Z \rightarrow(\tau \tau) Z$ & ATLAS & Figure $3 \mathrm{a}$ of [98] & $220-1000$ \\
\hline$g g \rightarrow A \rightarrow h Z \rightarrow(b \bar{b}) Z$ & ATLAS & Figure $3 \mathrm{~b}$ of [98] & $220-1000$ \\
\hline$p p \rightarrow A \rightarrow Z \gamma \rightarrow(\ell \ell) \gamma$ & CMS & Figure 2 of [99] & $200-1200$ \\
\hline
\end{tabular}

Table 4. The exclusion (upper) limits at $95 \%$ CL on the production cross-section times branching ratio of the processes considered in the $H$ and $A$ searches. The first four exclusion limits are employed in both, $H$ and $A$ searches.

\begin{tabular}{|c|c|c|}
\hline Observable & Value & Source \\
\hline$\Delta m_{B_{s}}$ & $17.757 \pm 0.021 \mathrm{ps}^{-1}$ & {$[100]$} \\
\hline $\mathcal{B}\left(\bar{B} \rightarrow X_{s} \gamma\right)$ & $3.43 \cdot 10^{-4} \pm 0.21 \cdot 10^{-4} \pm 0.07 \cdot 10^{-4}$ & {$[100]$} \\
\hline
\end{tabular}

Table 5. Flavour inputs.

\footnotetext{
${ }^{3}$ Signal strength (normalized to the SM expectation).
} 
Open Access. This article is distributed under the terms of the Creative Commons Attribution License (CC-BY 4.0), which permits any use, distribution and reproduction in any medium, provided the original author(s) and source are credited.

\section{References}

[1] ATLAS collaboration, Observation of a new particle in the search for the Standard Model Higgs boson with the ATLAS detector at the LHC, Phys. Lett. B 716 (2012) 1 [arXiv: 1207.7214] [INSPIRE].

[2] CMS collaboration, Observation of a new boson at a mass of $125 \mathrm{GeV}$ with the CMS experiment at the LHC, Phys. Lett. B 716 (2012) 30 [arXiv:1207.7235] [INSPIRE].

[3] ATLAS and CMS collaborations, Combined measurement of the Higgs boson mass in pp collisions at $\sqrt{s}=7$ and $8 \mathrm{TeV}$ with the ATLAS and CMS experiments, Phys. Rev. Lett. 114 (2015) 191803 [arXiv: 1503.07589] [INSPIRE].

[4] G. Degrassi et al., Higgs mass and vacuum stability in the Standard Model at NNLO, JHEP 08 (2012) 098 [arXiv: 1205.6497] [INSPIRE].

[5] D. Buttazzo et al., Investigating the near-criticality of the Higgs boson, JHEP 12 (2013) 089 [arXiv: 1307.3536] [INSPIRE].

[6] R. Barbieri and G.F. Giudice, Upper bounds on supersymmetric particle masses, Nucl. Phys. B 306 (1988) 63 [INSPIRE].

[7] M. Drees, R. Godbole and P. Roy, Theory and phenomenology of sparticles: an account of four-dimensional $N=1$ supersymmetry in high energy physics, World Scientific, Singapore (2004) [INSPIRE].

[8] H. Baer and X. Tata, Weak scale supersymmetry: from superfields to scattering events, Cambridge University Press, Cambridge U.K. (2006).

[9] S.P. Martin, A supersymmetry primer, Adv. Ser. Direct. High Energy Phys. 18 (1998) 1 [Adv. Ser. Direct. High Energy Phys. 21 (2010) 1] [hep-ph/9709356] [INSPIRE].

[10] G. Burdman, Z. Chacko, R. Harnik, L. de Lima and C.B. Verhaaren, Colorless top partners, a $125 \mathrm{GeV}$ Higgs and the limits on naturalness, Phys. Rev. D 91 (2015) 055007 [arXiv:1411.3310] [INSPIRE].

[11] G. Burdman, Z. Chacko, H.-S. Goh, R. Harnik and C.A. Krenke, The quirky collider signals of folded supersymmetry, Phys. Rev. D 78 (2008) 075028 [arXiv: 0805.4667] [INSPIRE].

[12] G. Burdman, Z. Chacko, H.-S. Goh and R. Harnik, Folded supersymmetry and the LEP paradox, JHEP 02 (2007) 009 [hep-ph/0609152] [INSPIRE].

[13] Z. Chacko, H.-S. Goh and R. Harnik, A twin Higgs model from left-right symmetry, JHEP 01 (2006) 108 [hep-ph/0512088] [INSPIRE].

[14] Z. Chacko, H.-S. Goh and R. Harnik, The twin Higgs: natural electroweak breaking from mirror symmetry, Phys. Rev. Lett. 96 (2006) 231802 [hep-ph/0506256] [INSPIRE].

[15] G. Panico and A. Wulzer, The composite Nambu-Goldstone Higgs, Lect. Notes Phys. 913 (2016) pp.1-316 [arXiv:1506.01961] [INSPIRE].

[16] K. Agashe, R. Contino and A. Pomarol, The minimal composite Higgs model, Nucl. Phys. B 719 (2005) 165 [hep-ph/0412089] [INSPIRE]. 
[17] ATLAS and CMS collaborations, Measurements of the Higgs boson production and decay rates and constraints on its couplings from a combined ATLAS and CMS analysis of the LHC pp collision data at $\sqrt{s}=7$ and 8 TeV, JHEP 08 (2016) 045 [arXiv:1606.02266] [INSPIRE].

[18] T.D. Lee, A theory of spontaneous T violation, Phys. Rev. D 8 (1973) 1226 [InSPIRE].

[19] J.F. Gunion and H.E. Haber, The CP conserving two Higgs doublet model: the approach to the decoupling limit, Phys. Rev. D 67 (2003) 075019 [hep-ph/0207010] [INSPIRE].

[20] G.C. Branco, P.M. Ferreira, L. Lavoura, M.N. Rebelo, M. Sher and J.P. Silva, Theory and phenomenology of two-Higgs-doublet models, Phys. Rept. 516 (2012) 1 [arXiv:1106.0034] [INSPIRE].

[21] A.E. Carcamo Hernandez, R. Martinez and J.A. Rodriguez, Different kind of textures of Yukawa coupling matrices in the two Higgs doublet model type-III, Eur. Phys. J. C 50 (2007) 935 [hep-ph/0606190] [INSPIRE].

[22] C.-Y. Chen and S. Dawson, Exploring two Higgs doublet models through Higgs production, Phys. Rev. D 87 (2013) 055016 [arXiv:1301.0309] [INSPIRE].

[23] C.-W. Chiang and K. Yagyu, Implications of Higgs boson search data on the two-Higgs doublet models with a softly broken $Z_{2}$ symmetry, JHEP 07 (2013) 160 [arXiv:1303.0168] [INSPIRE].

[24] B. Grinstein and P. Uttayarat, Carving out parameter space in type-II two Higgs doublets model, JHEP 06 (2013) 094 [Erratum ibid. 09 (2013) 110] [arXiv:1304.0028] [INSPIRE].

[25] A. Barroso, P.M. Ferreira, R. Santos, M. Sher and J.P. Silva, $2 H D M$ at the LHC - the story so far, arXiv:1304.5225 [INSPIRE].

[26] B. Coleppa, F. Kling and S. Su, Constraining type II 2 HDM in light of LHC Higgs searches, JHEP 01 (2014) 161 [arXiv:1305.0002] [INSPIRE].

[27] O. Eberhardt, U. Nierste and M. Wiebusch, Status of the two-Higgs-doublet model of type-II, JHEP 07 (2013) 118 [arXiv: 1305.1649] [INSPIRE].

[28] G. Bélanger, B. Dumont, U. Ellwanger, J.F. Gunion and S. Kraml, Global fit to Higgs signal strengths and couplings and implications for extended Higgs sectors, Phys. Rev. D 88 (2013) 075008 [arXiv:1306.2941] [inSPIRE].

[29] G. Bhattacharyya, D. Das, P.B. Pal and M.N. Rebelo, Scalar sector properties of two-Higgs-doublet models with a global U(1) symmetry, JHEP 10 (2013) 081 [arXiv: 1308.4297] [INSPIRE].

[30] S. Chang, S.K. Kang, J.-P. Lee, K.Y. Lee, S.C. Park and J. Song, Two Higgs doublet models for the LHC Higgs boson data at $\sqrt{s}=7$ and 8 TeV, JHEP 09 (2014) 101 [arXiv:1310.3374] [INSPIRE].

[31] K. Cheung, J.S. Lee and P.-Y. Tseng, Higgcision in the two-Higgs doublet models, JHEP 01 (2014) 085 [arXiv: 1310.3937] [INSPIRE].

[32] A. Celis, V. Ilisie and A. Pich, Towards a general analysis of LHC data within two-Higgs-doublet models, JHEP 12 (2013) 095 [arXiv: 1310.7941] [INSPIRE].

[33] L. Wang and X.-F. Han, Status of the aligned two-Higgs-doublet model confronted with the Higgs data, JHEP 04 (2014) 128 [arXiv:1312.4759] [INSPIRE]. 
[34] J. Baglio, O. Eberhardt, U. Nierste and M. Wiebusch, Benchmarks for Higgs pair production and heavy Higgs boson searches in the two-Higgs-doublet model of type II, Phys. Rev. D 90 (2014) 015008 [arXiv: 1403.1264] [InSPIRE].

[35] S. Inoue, M.J. Ramsey-Musolf and Y. Zhang, CP-violating phenomenology of flavor conserving two Higgs doublet models, Phys. Rev. D 89 (2014) 115023 [arXiv:1403.4257] [INSPIRE].

[36] B. Dumont, J.F. Gunion, Y. Jiang and S. Kraml, Constraints on and future prospects for two-Higgs-doublet models in light of the LHC Higgs signal, Phys. Rev. D 90 (2014) 035021 [arXiv: 1405.3584] [INSPIRE].

[37] S. Kanemura, K. Tsumura, K. Yagyu and H. Yokoya, Fingerprinting nonminimal Higgs sectors, Phys. Rev. D 90 (2014) 075001 [arXiv: 1406.3294] [INSPIRE].

[38] P.M. Ferreira, R. Guedes, J.F. Gunion, H.E. Haber, M.O.P. Sampaio and R. Santos, The CP-conserving 2HDM after the $8 \mathrm{TeV}$ run, arXiv:1407.4396 [INSPIRE].

[39] P.S. Bhupal Dev and A. Pilaftsis, Maximally symmetric two Higgs doublet model with natural Standard Model alignment, JHEP 12 (2014) 024 [Erratum ibid. 11 (2015) 147] [arXiv: 1408.3405] [INSPIRE].

[40] A. Broggio, E.J. Chun, M. Passera, K.M. Patel and S.K. Vempati, Limiting two-Higgs-doublet models, JHEP 11 (2014) 058 [arXiv:1409.3199] [INSPIRE].

[41] B. Dumont, J.F. Gunion, Y. Jiang and S. Kraml, Addendum to "constraints on and future prospects for two-Higgs-doublet models in light of the LHC Higgs signal", arXiv:1409.4088 [INSPIRE].

[42] J. Bernon, J.F. Gunion, Y. Jiang and S. Kraml, Light Higgs bosons in two-Higgs-doublet models, Phys. Rev. D 91 (2015) 075019 [arXiv:1412.3385] [INSPIRE].

[43] C.-Y. Chen, S. Dawson and Y. Zhang, Complementarity of LHC and EDMs for exploring Higgs CP-violation, JHEP 06 (2015) 056 [arXiv: 1503.01114] [INSPIRE].

[44] D. Chowdhury and O. Eberhardt, Global fits of the two-loop renormalized two-Higgs-doublet model with soft $Z_{2}$ breaking, JHEP 11 (2015) 052 [arXiv:1503.08216] [INSPIRE].

[45] N. Craig, F. D'Eramo, P. Draper, S. Thomas and H. Zhang, The hunt for the rest of the Higgs bosons, JHEP 06 (2015) 137 [arXiv: 1504.04630] [INSPIRE].

[46] J. Bernon, J.F. Gunion, H.E. Haber, Y. Jiang and S. Kraml, Scrutinizing the alignment limit in two-Higgs-doublet models: $m_{h}=125$ GeV, Phys. Rev. D 92 (2015) 075004 [arXiv: 1507.00933] [INSPIRE].

[47] J. Bernon, J.F. Gunion, H.E. Haber, Y. Jiang and S. Kraml, Scrutinizing the alignment limit in two-Higgs-doublet models. II. $m_{H}=125 \mathrm{GeV}$, Phys. Rev. D 93 (2016) 035027 [arXiv: 1511.03682] [INSPIRE].

[48] B.W. Lee, C. Quigg and H.B. Thacker, The strength of weak interactions at very high-energies and the Higgs boson mass, Phys. Rev. Lett. 38 (1977) 883 [INSPIRE].

[49] B.W. Lee, C. Quigg and H.B. Thacker, Weak interactions at very high-energies: the role of the Higgs boson mass, Phys. Rev. D 16 (1977) 1519 [INSPIRE].

[50] R. Casalbuoni, D. Dominici, R. Gatto and C. Giunti, Strong interacting two doublet and doublet singlet Higgs models, Phys. Lett. B 178 (1986) 235 [INSPIRE]. 
[51] J. Maalampi, J. Sirkka and I. Vilja, Tree level unitarity and triviality bounds for two Higgs models, Phys. Lett. B 265 (1991) 371 [INSPIRE].

[52] S. Kanemura, T. Kubota and E. Takasugi, Lee-Quigg-Thacker bounds for Higgs boson masses in a two doublet model, Phys. Lett. B 313 (1993) 155 [hep-ph/9303263] [INSPIRE].

[53] A.G. Akeroyd, A. Arhrib and E.-M. Naimi, Note on tree level unitarity in the general two Higgs doublet model, Phys. Lett. B 490 (2000) 119 [hep-ph/0006035] [INSPIRE].

[54] I.F. Ginzburg and I.P. Ivanov, Tree-level unitarity constraints in the most general 2HDM, Phys. Rev. D 72 (2005) 115010 [hep-ph/0508020] [INSPIRE].

[55] J. Horejsi and M. Kladiva, Tree-unitarity bounds for THDM Higgs masses revisited, Eur. Phys. J. C 46 (2006) 81 [hep-ph/0510154] [InSPIRE].

[56] H.E. Haber and D. O'Neil, Basis-independent methods for the two-Higgs-doublet model III: the CP-conserving limit, custodial symmetry and the oblique parameters $S, T, U$, Phys. Rev. D 83 (2011) 055017 [arXiv: 1011.6188] [INSPIRE].

[57] B. Grinstein, C.W. Murphy and P. Uttayarat, One-loop corrections to the perturbative unitarity bounds in the CP-conserving two-Higgs doublet model with a softly broken $Z_{2}$ symmetry, JHEP 06 (2016) 070 [arXiv:1512.04567] [INSPIRE].

[58] L. Durand, J.M. Johnson and J.L. Lopez, Perturbative unitarity and high-energy $W_{L}^{ \pm}, Z_{L}$, $H$ scattering. One loop corrections and the Higgs boson coupling, Phys. Rev. D 45 (1992) 3112 [INSPIRE].

[59] P.N. Maher, L. Durand and K. Riesselmann, Two loop renormalization constants and high-energy $2 \rightarrow 2$ scattering amplitudes in the Higgs sector of the Standard Model, Phys. Rev. D 48 (1993) 1061 [Erratum ibid. D 52 (1995) 553] [hep-ph/9303233] [INSPIRE].

[60] L. Durand, P.N. Maher and K. Riesselmann, Two loop unitarity constraints on the Higgs boson coupling, Phys. Rev. D 48 (1993) 1084 [hep-ph/9303234] [INSPIRE].

[61] U. Nierste and K. Riesselmann, Higgs sector renormalization group in the MS and OMS scheme: the breakdown of perturbation theory for a heavy Higgs, Phys. Rev. D 53 (1996) 6638 [hep-ph/9511407] [INSPIRE].

[62] A. Delgado, G. Nardini and M. Quirós, A light supersymmetric Higgs sector hidden by a Standard Model-like Higgs, JHEP 07 (2013) 054 [arXiv: 1303.0800] [INSPIRE].

[63] N. Craig, J. Galloway and S. Thomas, Searching for signs of the second Higgs doublet, arXiv: 1305.2424 [INSPIRE].

[64] M. Carena, I. Low, N.R. Shah and C.E.M. Wagner, Impersonating the Standard Model Higgs boson: alignment without decoupling, JHEP 04 (2014) 015 [arXiv: 1310.2248] [INSPIRE].

[65] N.G. Deshpande and E. Ma, Pattern of symmetry breaking with two Higgs doublets, Phys. Rev. D 18 (1978) 2574 [INSPIRE].

[66] A. Barroso, P.M. Ferreira, I.P. Ivanov and R. Santos, Metastability bounds on the two Higgs doublet model, JHEP 06 (2013) 045 [arXiv: 1303.5098] [INSPIRE].

[67] H.E. Haber, Introductory low-energy supersymmetry, in Theoretical Advanced Study Institute (TASI 92): from black holes and strings to particles, Boulder U.S.A. June 3-28 1992 [hep-ph/9306207] [INSPIRE].

[68] C.Q. Geng and J.N. Ng, Charged Higgs effect in $B_{d}^{0}-\bar{B}_{d}^{0}$ mixing, $K \rightarrow \pi \nu \bar{\nu}$ decay and rare decays of B mesons, Phys. Rev. D 38 (1988) 2857 [Erratum ibid. D 41 (1990) 1715] [INSPIRE]. 
[69] O. Deschamps, S. Descotes-Genon, S. Monteil, V. Niess, S. T'Jampens and V. Tisserand, The two Higgs doublet of type II facing flavour physics data, Phys. Rev. D 82 (2010) 073012 [arXiv: 0907.5135] [INSPIRE].

[70] M. Misiak et al., Updated NNLO QCD predictions for the weak radiative B-meson decays, Phys. Rev. Lett. 114 (2015) 221801 [arXiv:1503.01789] [INSPIRE].

[71] J. de Blas et al., Electroweak precision observables and Higgs-boson signal strengths in the Standard Model and beyond: present and future, arXiv:1608.01509 [INSPIRE].

[72] LHC Higgs Cross section Working Group collaboration, J.R. Andersen et al., Handbook of LHC Higgs cross sections: 3. Higgs properties, arXiv:1307.1347 [INSPIRE].

[73] A. Djouadi, J. Kalinowski and M. Spira, HDECAY: a program for Higgs boson decays in the Standard Model and its supersymmetric extension, Comput. Phys. Commun. 108 (1998) 56 [hep-ph/9704448] [INSPIRE].

[74] J.F. Gunion, H.E. Haber, G.L. Kane and S. Dawson, The Higgs hunter's guide, Front. Phys. 80 (2000) 1 [INSPIRE].

[75] LHC Higgs Cross section Working Group collaboration, LHC Higgs Cross Section Working Group webpage, https://twiki.cern.ch/twiki/bin/view/LHCPhysics/LHCHXSWG, (2015).

[76] M. Spira, HIGLU: a program for the calculation of the total Higgs production cross-section at hadron colliders via gluon fusion including QCD corrections, hep-ph/9510347 [INSPIRE].

[77] R.V. Harlander, S. Liebler and H. Mantler, SusHi: a program for the calculation of Higgs production in gluon fusion and bottom-quark annihilation in the Standard Model and the MSSM, Comput. Phys. Commun. 184 (2013) 1605 [arXiv:1212.3249] [INSPIRE].

[78] J. Alwall et al., The automated computation of tree-level and next-to-leading order differential cross sections and their matching to parton shower simulations, JHEP $\mathbf{0 7}$ (2014) 079 [arXiv: 1405.0301] [INSPIRE].

[79] Particle Data Group collaboration, K.A. Olive et al., Review of particle physics, Chin. Phys. C 38 (2014) 090001 [INSPIRE].

[80] HEPFIT collaboration, HEPfit: a code for the combination of indirect and direct constraints on high energy physics models, http://hepfit.roma1.infn.it, in preparation.

[81] A. Caldwell, D. Kollar and K. Kroninger, BAT: the Bayesian Analysis Toolkit, Comput. Phys. Commun. 180 (2009) 2197 [arXiv:0808.2552] [InSPIRE].

[82] G.C. Branco, L. Lavoura and J.P. Silva, CP violation, Int. Ser. Monogr. Phys. 103 (1999) 1 [INSPIRE].

[83] S. Davidson and H.E. Haber, Basis-independent methods for the two-Higgs-doublet model, Phys. Rev. D 72 (2005) 035004 [Erratum ibid. D 72 (2005) 099902] [hep-ph/0504050] [INSPIRE].

[84] ATLAS collaboration, Search for neutral Higgs bosons of the minimal supersymmetric Standard Model in pp collisions at $\sqrt{s}=8 \mathrm{TeV}$ with the ATLAS detector, JHEP 11 (2014) 056 [arXiv: 1409.6064] [INSPIRE].

[85] CMS collaboration, Search for additional neutral Higgs bosons decaying to a pair of tau leptons in pp collisions at $\sqrt{s}=7$ and 8 TeV, CMS-PAS-HIG-14-029, CERN, Geneva Switzerland (2014). 
[86] ATLAS collaboration, Search for scalar diphoton resonances in the mass range $65-600 \mathrm{GeV}$ with the ATLAS detector in pp collision data at $\sqrt{s}=8$ TeV, Phys. Rev. Lett. 113 (2014) 171801 [arXiv: 1407.6583] [INSPIRE].

[87] CMS collaboration, Search for diphoton resonances in the mass range from 150 to $850 \mathrm{GeV}$ in pp collisions at $\sqrt{s}=8 \mathrm{TeV}$, Phys. Lett. B 750 (2015) 494 [arXiv: 1506. 02301] [INSPIRE].

[88] CMS collaboration, Search for neutral MSSM Higgs bosons decaying into a pair of bottom quarks, JHEP 11 (2015) 071 [arXiv:1506.08329] [INSPIRE].

[89] ATLAS collaboration, Search for a high-mass Higgs boson decaying to a $W$ boson pair in pp collisions at $\sqrt{s}=8 \mathrm{TeV}$ with the ATLAS detector, JHEP 01 (2016) 032 [arXiv: 1509.00389] [INSPIRE].

[90] ATLAS collaboration, Search for an additional, heavy Higgs boson in the $H \rightarrow Z Z$ decay channel at $\sqrt{s}=8 \mathrm{TeV}$ in pp collision data with the ATLAS detector, Eur. Phys. J. C 76 (2016) 45 [arXiv: 1507. 05930] [INSPIRE].

[91] CMS collaboration, Search for a Higgs boson in the mass range from 145 to $1000 \mathrm{GeV}$ decaying to a pair of $W$ or $Z$ bosons, JHEP 10 (2015) 144 [arXiv:1504.00936] [INSPIRE].

[92] ATLAS collaboration, Searches for Higgs boson pair production in the $h h \rightarrow b b \tau \tau, \gamma \gamma W W^{*}, \gamma \gamma b b, b b b b$ channels with the ATLAS detector, Phys. Rev. D 92 (2015) 092004 [arXiv: 1509.04670] [INSPIRE].

[93] CMS collaboration, Model independent search for Higgs boson pair production in the $b \bar{b} \tau^{+} \tau^{-}$final state, CMS-PAS-HIG-15-013, CERN, Geneva Switzerland (2015).

[94] CMS collaboration, Search for two Higgs bosons in final states containing two photons and two bottom quarks in proton-proton collisions at 8 TeV, Phys. Rev. D 94 (2016) 052012 [arXiv: 1603.06896] [INSPIRE].

[95] CMS collaboration, Search for resonant pair production of Higgs bosons decaying to two bottom quark-antiquark pairs in proton-proton collisions at 8 TeV, Phys. Lett. B 749 (2015) 560 [arXiv: 1503.04114] [INSPIRE].

[96] CMS collaboration, Searches for a heavy scalar boson $H$ decaying to a pair of $125 \mathrm{GeV}$ Higgs bosons hh or for a heavy pseudoscalar boson A decaying to $Z$ h, in the final states with $h \rightarrow \tau \tau$, Phys. Lett. B 755 (2016) 217 [arXiv:1510.01181] [INSPIRE].

[97] CMS collaboration, Search for a pseudoscalar boson decaying into a $Z$ boson and the $125 \mathrm{GeV}$ Higgs boson in $\ell^{+} \ell^{-} b \bar{b}$ final states, Phys. Lett. B 748 (2015) 221 [arXiv: 1504.04710] [INSPIRE].

[98] ATLAS collaboration, Search for a CP-odd Higgs boson decaying to Zh in pp collisions at $\sqrt{s}=8 \mathrm{TeV}$ with the ATLAS detector, Phys. Lett. B 744 (2015) 163 [arXiv:1502.04478] [INSPIRE].

[99] CMS collaboration, Search for scalar resonances in the 200-1200 GeV mass range decaying into $a \mathrm{Z}$ and a photon in pp collisions at $\sqrt{s}=8 \mathrm{TeV}$, CMS-PAS-HIG-16-014, CERN, Geneva Switzerland (2016).

[100] Heavy Flavor Averaging Group (HFAG) collaboration, Y. Amhis et al., Averages of b-hadron, c-hadron and $\tau$-lepton properties as of summer 2014, arXiv:1412.7515 [INSPIRE]. 